\title{
Cis-regulatory Element Hijacking by Structural Variants Overshadows Higher-Order Topological Changes in Prostate Cancer
}

James R. Hawley ${ }^{1,2, *}$, Stanley Zhou ${ }^{1,2, \$}$, Christopher Arlidge ${ }^{1}$, Giacomo Grillo ${ }^{1}$, Ken Kron 1, Rupert Hugh-White ${ }^{8,9,10}$, Theodorus van der Kwast ${ }^{3}$, Michael Fraser ${ }^{1,4}$, Paul C. Boutros ${ }^{2}$, $6,7,8,9,10$, Robert G. Bristow ${ }^{1,2,11-14}$, Mathieu Lupien ${ }^{1,2,5, *}$

1. Princess Margaret Cancer Centre, University Health Network, Toronto, Canada

2. Department of Medical Biophysics, University of Toronto, Toronto, Canada

3. Department of Laboratory Medicine and Pathobiology, University of Toronto, Toronto, Canada

4. Department of Surgery (Urology), University of Toronto, Toronto, Canada

5. Ontario Institute for Cancer Research, Toronto, Ontario, Canada

6. Department of Pharmacology and Toxicology, University of Toronto, Toronto, Canada

7. Department of Human Genetics, University of California, Los Angeles, USA

8. Department of Urology, University of California, Los Angeles, USA

9. Institute for Precision Health, University of California, Los Angeles, USA

10. Jonsson Comprehensive Cancer Center, University of California, Los Angeles, USA

11. Department of Radiation Oncology, University of Toronto, Toronto, Canada

12. CRUK Manchester Institute and Manchester Cancer Research Centre, Manchester,

$\mathrm{UK}$

13. Division of Cancer Sciences, Faculty of Biology, Health and Medicine, University of Manchester, Manchester, UK

14. The Christie NHS Foundation Trust, Manchester, UK

$\$$ The authors co-led the study with equal contributions and can be interchangeably listed as first author.

* Corresponding author: Mathieu Lupien, mlupien@uhnresearch.ca

Keywords: prostate, cancer, topologically associating domains, genetic architecture, three-dimensional genome, CTCF, chromatin interaction, loop, structural variants, mutations, noncoding, cis-regulatory elements, $\mathrm{H} 3 \mathrm{~K} 27 \mathrm{ac}, \mathrm{Hi}-\mathrm{C}$, chromatin, epigenetics. 


\section{Abstract}

Prostate cancer is a heterogeneous disease whose progression is linked to genome instability. However the impact of this instability on the three-dimensional chromatin organization and how this drives progression is unclear. Using primary benign and tumour tissue, we find a high concordance in the higher-order three-dimensional genome organization across normal and prostate cancer cells. This concordance argues for constraints to the topology of prostate tumour genomes. Nonetheless, we identify changes to focal chromatin interactions and show how structural variants can induce these changes to guide cis-regulatory element hijacking. Such events result in opposing differential expression on genes found at antipodes of rearrangements. Collectively, our results argue that cis-regulatory element hijacking from structural variant-induced altered focal chromatin interactions overshadows higher-order topological changes in the development of primary prostate cancer. 


\section{Introduction}

The human genome is organized into hubs of chromatin interactions within the nucleus, setting its three-dimensional topology ${ }^{1}$. Two classes of higher-order topology, topologically associating domains (TADs) and compartments, define clusters of contacts between DNA elements that are linearly distant from each other, such as cis-regulatory elements (CREs) and their target gene promoters ${ }^{2,3}$. Insulating these hubs to prevent ectopic interactions are TAD boundaries, maintained by CCCTC-binding Factor (CTCF) and the cohesin complex ${ }^{4}$. Disruption of TAD boundaries through genetic or epigenetic variants can activate oncogenes, as observed in medulloblastoma ${ }^{5}$, acute myeloid leukemia ${ }^{6}$, gliomas ${ }^{7}$, and salivary gland acinic cell carcinoma ${ }^{8}$. However, recent studies depleting CTCF or the cohesin complex produced little effect on gene expression despite global changes to the three-dimensional chromatin organization ${ }^{9-11}$. In contrast, CRE hijacking caused by genetic alterations can result in large changes to gene expression, despite having little impact on the higher-order chromatin organization ${ }^{5,12}$. These contrasting observations raise questions about the interplay between components of the genetic architecture, namely, how genetic alterations, chromatin states, and the three-dimensional genome cooperate to misregulate genes in disease. Understanding the roles that chromatin organization and cis-regulatory interactions play in gene regulation is crucial for understanding how their disruption can promote oncogenesis.

The roles of noncoding mutations targeting CREs in cancer are becoming increasingly clear ${ }^{12-14}$. Mutations to the TERT promoter, for example, lead to its over-expression and telomere elongation in multiple cancer types ${ }^{15-17}$. Similarly, mutations targeting CREs of the 
ESR1 and FOXA1 oncogenes in breast and prostate cancers, respectively, lead to their sustained over-expression ${ }^{18-20}$, which is associated with resistance to hormonal therapies ${ }^{21-24}$. Point mutations have the potential to alter three-dimensional chromatin organization, albeit indirectly, by modifying transcription factor or CTCF binding sites ${ }^{25,26}$. Structural variants (SVs), on the other hand, are large rearrangements of chromatin that can directly impact its structure ${ }^{27,28}$. This can establish novel CRE interactions from separate TADs or chromosomes, as has been observed in leukemia ${ }^{29}$ and multiple developmental diseases ${ }^{30,31}$. But how prevalent and to what extent these rearrangements affect the surrounding chromatin remains largely unstudied in primary tumours ${ }^{14,28,32}$. Hence, to understand gene misregulation in cancer, it is critical to understand how SVs impact three-dimensional chromatin organization and CRE interactions in primary tumours.

SVs play an important role in prostate cancer (PCa), both for oncogenesis and progression. An estimated $97 \%$ of primary tumours contain SVs ${ }^{14,33}$, and translocations and duplications of CREs for oncogenes such as $A R^{34}, E R G^{35}, F O X A 1^{20,36}$ and $M Y C{ }^{20}$ are highly recurrent. While coding mutations of FOXA1 are found in $\sim 10 \%$ of metastatic castration-resistant PCa patients, SVs that target FOXA1 CREs are found in over $25 \%$ of metastatic prostate tumours ${ }^{20}$. In addition to oncogenic activation, SVs in prostate tumours disrupt and inactivate key tumour suppressor genes including PTEN, BRCA2, CDK12, and TP53 ${ }^{36,37}$. Furthermore, over $90 \%$ of prostate tumours contain complex SVs, including chromothripsis and chromoplexy events ${ }^{38}$, making it a prime model to study the effects of SVs. However, despite large-scale tumour sequencing efforts, investigating the impact of SVs on three-dimensional prostate genome remains difficult, owing to constraints from chromatin conformation capture (i.e. Hi-C) assays. In this work, we build on recent technological advances in $\mathrm{Hi}-\mathrm{C}$ protocols to investigate the three-dimensional chromatin organization of the 
prostate from primary benign and tumour tissues. Using patient-matched whole genome sequencing (WGS), RNA sequencing (RNA-seq), and chromatin immunoprecipitation (ChIP-seq) data, we show that SVs in PCa repeatedly hijacking CREs to disrupt the expression of multiple genes with minimal impact to higher-order three-dimensional chromatin organization. 


\section{Results}

\section{Three-dimensional Chromatin organization is stable over oncogenesis}

Chromatin conformation capture technologies enable the measurement of three-dimensional chromatin organization. These assays, however, are often limited to cell lines, animal models and liquid tumours due to the amount of input required ${ }^{39}$. Here, we optimized and conducted low-input $\mathrm{Hi}-\mathrm{C}{ }^{40}$ on $10 \mu \mathrm{m}$ thick cryosections from 12 primary prostate tumours and 5 primary benign prostate sections (see Methods, Figure 1a, Supplementary Figure 1a). The 12 tumours were selected from the Canadian Prostate Cancer Genome Network (CPC-GENE) cohort previously assessed for whole-genome sequencing ${ }^{33}$, RNA-seq ${ }^{41}$ and H3K27ac ChIP-seq ${ }^{42,43}$ (Supplementary Table 1). All 12 of these PCa patients previously underwent radical prostatectomies and 6 of our 12 samples $(50 \%)$ harbour the TMPRSS2-ERG genetic fusion (T2E) found in approximately half of the primary PCa patients ${ }^{33}$. The total percent of genome altered ranges from $0.99 \%-18.78 \%$ (Supplementary Table 1) ${ }^{33}$. The 12 tumour samples were histopathologically assessed to have $\geq 70 \%$ cellularity while the cellularity was $\geq 60 \%$ for our group of 5 normal prostate samples. Upon Hi-C sequencing, we reached an average of $9.90 \times 10^{8}$ read pairs per sample (range $5.84 \mathrm{x}$ $10^{8}-1.49 \times 10^{9}$ read pairs) with minimal duplication rates (range $10.6 \%-20.8 \%$ ) (Supplementary Table 2). Pre-processing resulted in an average of $6.23 \times 10^{8}(96.13 \%)$ valid read pairs per sample (range $3.95 \times 10^{8}-9.01 \times 10^{8}$, or $82.42-99.22 \%$; Supplementary Table 2). Hence, we produced a high depth, high quality Hi-C library on 17 primary prostate tissue slices. 
To characterize the higher-order organization of the primary prostate genome, we first identified TADs. Across the 17 primary tissue samples, we observed an average of 2,305 TADs with a median size of $560 \mathrm{kbp}$ (Supplementary Tables 3-4). However, when considering all hierarchical levels of TAD organization, we did not observe significant differences in the number of TADs identified across length scales (Figure 1b), nor in the persistence of their boundaries (Figure 1c). This suggests few, if any, differences in three-dimensional chromatin organization at the TAD level between benign and tumour tissue. However, we observed differences in organization around essential genes for $\mathrm{PCa}$ between primary tissue and previously profiled cell lines. For example, chromatin around the $A R$ gene that was previously found enriched in the 22Rv1 compared to RWPE1 prostate cell lines ${ }^{44}$ were not recapitulated in either benign or tumour primary samples (Figure 1d, Supplementary Figure 1d). Moreover, when compared to other Hi-C datasets, the primary prostate samples clustered separately from cell lines (Supplementary Figure 1b), despite similar enrichment of CTCF binding sites near TAD boundaries (Supplementary Figure 1c). These results suggest that TADs are constrained over oncogenesis and that cell line models may not harbour disease-relevant three-dimensional chromatin organization.

We next investigated compartmentalization changes, the second class of higher-order three-dimensional chromatin organization. Recurrent changes to segments nearly the size of chromosome arms showed differential compartmentalization in multiple tumour samples compared to benign samples, such as compartment B-to-A transitions on $19 \mathrm{q}$ and A-to-B transitions on chromosome Y (Supplementary Figure 2a-c). Only two genes on chromosome 19 were differentially expressed between the 8 tumours with benign-like compartmentalization and the other 4 (Supplementary Figure 2d). Similarly, no genes on chromosome $\mathrm{Y}$ were differentially expressed between the 4 tumours with benign-like 
compartmentalization and the remaining samples (Supplementary Figure 2e). Both arms on chromosome 3 show differential mean compartmentalization, but this appears to be driven by one tumour sample and one benign sample for each arm and is not recurrent (Supplementary Figure 2f). Collectively, these results suggest that phenotypic differences between benign and tumour tissues do not stem from differences in higher-order three-dimensional chromatin organization alone.

\section{Focal chromatin interactions shifting over oncogenesis}

Changes to focal chromatin interactions have been observed in the absence of higher-order chromatin changes ${ }^{45,46}$, and we hypothesized that this may be the case in PCa. We detected chromatin interactions, identifying a median of 4,395 interactions per sample (range 1,286 - 6,993; Supplementary Figure 3a, Supplementary Table 5). Among these detected interactions, we identified known contacts in PCa such as those between two distal CREs on chromosome 14 and the FOXA1 promoter $^{19}$ (Supplementary Figure 3b), and CREs upstream of $M Y C$ on chromosome 8 that are frequently duplicated in metastatic disease ${ }^{36}$ (Supplementary Table 5). 16,474 unique chromatin interactions were identified in at least one sample (Figure 2a), reaching an estimated $\sim 80 \%$ saturation of detection (Supplementary Figure 3c). Restricting our analysis to the 8,486 interactions present in at least two samples (51.5\% of all interactions) yielded 1,405 tumour- and 273 benign-specific interactions, suggesting focal changes in three-dimensional chromatin organization occur over oncogenesis. Aggregate peak analysis revealed Hi-C contact enrichment at all detected interactions in all samples (Figure 2b-c), demonstrating that tumors- and benign-specific interactions are not binary. Rather, the contacts at "tumour-specific" loci are more enriched than those at "benign-specific" loci in tumour samples (Figure 2b). Similarly, the contacts at 
"benign-specific" loci are more enriched than those at "tumour-specific" loci in benign samples (Figure 2c). Together, these results suggest that more focal changes to chromatin interactions are present in prostate oncogenesis despite the stable higher-order organization.

\section{Cataloguing structural variants from $\mathrm{Hi}-\mathrm{C}$ data}

In prostate tumours, SVs populate the genome to aid disease onset and progression 33,36. Advances in computational methods now enable the identification of SVs from Hi-C datasets ${ }^{27,47}$. Applying an SV caller to our primary prostate tumour $\mathrm{Hi}-\mathrm{C}$ dataset $^{27}$, we detected a total of 317 unique breakpoints with a median of 15 unique breakpoints per tumour (range 3-95; Figure 3a; Supplementary Table 6). As an example, we found evidence of the TMPRSS2-ERG (T2E) genetic fusion spanning the 21q22.2-3 locus in 6/12 (50\%) patients (CPCG0258, CPCG0324, CPCG0331, CPCG0336, CPCG0342, and CPCG0366) (Figure 3b), in accordance with previous whole-genome sequencing (WGS) findings ${ }^{33}$. Combining unique breakpoint pairs into rearrangement events yielded 7.5 total events on average per patient (range 1 - 36, Supplementary Figure 4a-b). We also identified more inter-chromosomal breakpoint pairs with the Hi-C data in 11 of 12 tumours (Figure 3b), including a novel translocation event that encompasses the deleted region between TMPRSS2 and $E R G$ into chromosome 14. Few loci contained SV breakpoints recurrent between patients (Supplementary Figure 4c). These numbers are smaller than previously reported from matched WGS data ${ }^{33}$; however, the median distance between breakpoints on the same chromosome was much larger at 31.6 Mbp for Hi-C-identified breakpoints, compared to 1.47 Mbp from WGS-identified breakpoints (Figure 3c). This is consistent with the inherent nature and resolution of the Hi-C method to detect larger, inter-chromosomal events ${ }^{27}$. No SVs were detected in the 5 primary benign prostate tissue samples from Hi-C data. While this does not 
rule out the presence of small rearrangements undetectable by Hi-C limited by its resolution, the absence of large and inter-chromosomal SVs further supports a difference in genome stability between benign and tumour tissues ${ }^{33,38,43,48}$. Collectively, Hi-C defines a valid method to interrogate for the presence of SV in tumour samples, compatible with the detection of intra- and inter-chromosomal interactions otherwise missed in WGS analyses.

Among SVs detected in primary prostate tumours, we identified both simple and complex chains of breakpoints. While simple SVs correspond to fusion between two distal DNA sequences, complex chains are evidence of chromothripsis and chromoplexy ${ }^{38}$. These genomic aberrations affecting multiple regions of the genome are known to occur in both primary and metastatic $\mathrm{PCa}{ }^{14,33,38}$. The chains can be pictured as paths connecting breakpoints in the contact matrix (Supplementary Figure 4d). 8 of the $12(66.7 \%)$ tumour samples contained these chains, including one patient (CPCG0331) harbouring 11 complex events and three patients (CPCG0246, CPCG0345, and CPCG0365) each harbouring a single complex event. We observed a median of 1 complex event per patient (range $0-11)$ consisting of a median of 3 breakpoints (range 3-7) spanning a median of 2 chromosomes per event (range 1-4, Supplementary Table 7, Supplementary Figure 4b). Patient CPCG0331 had 11 complex events, including a 6-breakpoint event spanning 3 chromosomes (Supplementary Figure 4b). A highly rearranged chromosome 3 was also found in the same patient (Figure 3d). The most common type of complex event involved 3 breakpoints and spanned 2 chromosomes, occurring 9 times across 5 of the 8 patients with complex events. In summary, using Hi-C, we detected both simple and complex SVs in primary prostate tumours not previously identified using WGS-based methods. We were able to identify known observations, such as a highly mutated region on chromosome 3 and subtype-specific 
differences in abundance, as well as find novel inter-chromosomal events not previously reported.

\section{SVs alter gene expression independently of intra-TAD contacts}

Using combined WGS called SVs with those from Hi-C data, we next systematically examined the impact of SVs on TAD structure. This led us to look at the intra-TAD and inter-TAD interactions around each breakpoint. We observed that only 18 of the $260(6.9 \%)$ TADs containing SV breakpoints were associated with decreased intra-TAD or increased inter-TAD interactions (Figure 4a). 12 of $18(66.7 \%)$ occurrences were within T2E+ tumours. We found no evidence that simple versus complex SVs were a factor in determining whether a TAD was altered (Pearson's chi-square test, $\mathrm{X}^{2}=0.0166, \mathrm{p}=0.8974, \mathrm{df}=1$ ). Similarly, the type of SV (a deletion, inversion, duplication, or translocation) was not predictive of whether the TAD would be altered (Pearson's chi-square test, $\mathrm{X}^{2}=4.7756, \mathrm{p}=0.3111$, df $=4$ ). Overall, we find that SVs are associated with higher-order topological changes in a small percentage of cases and that the presence of an SV breakpoint is not predictive alone of an altered TAD.

Despite the evidence that SVs rarely impact higher-order chromatin topology, we evaluated whether SVs affected the expression of genes within the TADs surrounding the breakpoint using patient-matched RNA-seq data ${ }^{41}$. We found that 23 of 260 breakpoints $(8.8 \%)$ are associated with significant changes to local gene expression (Figure $4 \mathrm{~b}$ ). Complex events can have opposite effects at each breakpoint. For example, while the T2E fusion across all tumours leads to over-expression of ERG and under-expression of TMPRSS $2^{33,42}$, the deleted locus between these two genes was inserted into chromosome 14 as part of a complex translocation event in one patient (Figure 4c-f). This inserted fragment positions 
ERG towards the 5' end of the RALGAPA1 gene and TMPRSS2 towards the 3' end (Figure 4c) resulting in a significant drop in intra-TAD contacts at the RALGAPA1 locus on chromosome 14 (two-sample unpaired t-test, $\mathrm{t}=6.38, \mathrm{p}=1.04 \mathrm{e}-9$; Figure $4 \mathrm{~d}$ ). Despite the significant topological change on chromosome 14, no significant changes to expression was detectable across genes within the same TAD on chromosome 14 (Figure 4e). Conversely, TAD alterations are not required changes to gene expression. As part of a complex SV involving the $R I M B P 2$ gene (Figure $4 \mathrm{~g}-\mathrm{j}$ ), both ends of the gene contain breakpoints (Figure $4 \mathrm{~g}$ ). This rearrangement is not associated with changes to intra-TAD contacts (two-sample unpaired t-test, $\mathrm{t}=0.8101, \mathrm{p}=0.4183$; Figure $4 \mathrm{~h})$. However, RIMBP2 is over-expressed in this patient (Figure 4i). More generally, only a single breakpoint was observed with both TAD contact and gene expression changes, although we did not find evidence to suggest these are dependent events (Pearson's chi-square test, $\mathrm{X}^{2}=6.31 \mathrm{e}-3, \mathrm{p}=0.9367 \mathrm{df}=1$ ). For TADs where at least one gene was differentially expressed, $19(83 \%)$ of them had at least one gene with doubled or halved expression. Notably, we found that inter-chromosomal translocations are associated with altering the expression of genes nearby their breakpoints compared to intra-chromosomal breakpoints (Pearson's chi-squared test, $\mathrm{X}^{2}=7.0088, \mathrm{p}=$ $0.00811, \mathrm{df}=1$; Supplementary Figure 5). Taken together, these results suggest that while SVs can alter contacts within TADs, this is neither necessary nor sufficient to alter gene expression.

\section{SVS alter focal chromatin interactions to hijack CREs and alter antipode gene}

\section{expression}

Mutations in prostate cancer have previously been found to converge on active CREs

49. To assess if SVs function in a similar fashion, we investigated the convergence of SV 
breakpoints in active CREs. We find that SV breakpoints are enriched in the catalogue of CREs captured by H3K27ac ChIP-seq from our 12 primary prostate tumours compared to the rest of the genome (one-sided permutation $z$-test, $\mathrm{z}=25.591, \mathrm{p}=0.0099, \mathrm{n}=100$; Figure 5a-b). This is similar to the enrichment of point mutations in CREs active in prostate cancer ${ }^{49}$, suggesting that SVs which alter gene expression may do so by recurrently targeting CREs. Since individual CREs can regulate multiple genes ${ }^{50}$, we suspected that SVs that do alter gene expression may predominantly affect multiple genes at the same time, instead of single genes. In agreement, when considering all SVs associated with altered gene expression near a breakpoint we find 16 of the $22(72.7 \%)$ SVs are associated with altered expression of multiple genes (Figure 5c-d). Notably, 15 of these 16 SVs $(93.8 \%)$ are associated with both over- and under-expression of genes, instead of genes all being either over-expressed or under-expressed (Figure 5d). 12 of these 15 (80\%) SVs are associated with expression changes at SV antipodes, opposite ends of a breakpoint pair (Supplementary Figure 6). The recurrent targeting of active CREs, combined with the opposite gene expression changes at SV antipodes, suggests that SVs may repeatedly alter expression by CRE hijacking.

The fusion of PMEPA1 and ZNF516 is an example of CRE hijacking resulting in opposite differential gene expression. Specifically, the fusion results in the PMEPA1 promoter being hijacked to the 5' end of the ZNF516 gene. This is concomitant with the over-expression of ZNF516 and under-expression of PMEPA1 (Figure 6a-c). In addition to hijacking the PMEPA1 promoter to the ZNF516 gene, this fusion also coincides with gains in H3K27ac over the ZNF516 gene body and of H3K27ac histone hypoacetylation over the 3' end of PMEPAI's gene body. This mirrors the creation of a Cluster Of Regulatory Elements (COREs) reported for the T2E fusion, reflective of new CREs enabling $E R G$ over-expression and the concomitant under-expression of TMPRSS2 (Supplementary Figure 7) ${ }^{42,51,52}$. CRE 
hijacking is also observed with inter-chromosomal rearrangements such as seen at the SV connecting chromosomes 7 and 19, creating 2 fusion products (termed C2B and B2C; Figure 6d). This SV separates the 3' end of $B R A F$ from its promoter and upstream enhancers on chromosome 7 (C2B; Figure 6d), fusing it to the 3' end of CYP4F11 (Figure 6e). Focal chromatin interactions between $B R A F$ and multiple active CREs are only observed in the fusion on chromosome 19 (Figure 6e). Using matched RNA-seq data, we observe an estimated 5 fold increase in expression for the 3' exons of $B R A F$ in the mutated tumour compared to others (fold-change $=4.976$, FDR $=0.0181$; Figure 6f). Collectively, over-expression of the oncogenes, such as $E R G$ and $B R A F$, and suppression of the tumour suppressor PMEPA1 demonstrates the disease-relevant effects of CRE hijacking mediated by SVs in primary prostate cancer resulting in changes to focal chromatin interactions, and that these effects overshadow the effect on higher-order topology in primary prostate cancer. 


\section{Discussion}

Genetic alterations that subvert the higher-order chromatin organization to allow for aberrant focal interactions may be more common in cancer than previously recognized. In this work we demonstrated that CRE hijacking by SVs is often associated with opposing gene expression changes at SV antipodes, whereby genes on one flank of the breakpoint are upregulated while genes on the other flank are repressed. Complex SVs, such as chromoplexy and chromothripsis, are found in numerous cancer types ${ }^{14,38}$, providing many opportunities for widespread effects on gene expression and CRE hijacking. This is in addition to many known cancer drivers that alter CRE interactions, including the $A R$ and FOXA1 enhancer amplifications in primary and metastatic prostate tumours ${ }^{19,20,34,36,42,53}$. More recent findings also fit this model, such as accumulation of extrachromosomal circular DNA activating oncogenes that would otherwise be constrained by chromatin topology ${ }^{54-57}$. These insights stress the importance of investigating all ends of an SV to assess the biological impact of these mutations on the cis-regulatory landscape as a whole, as opposed to focusing on CREs or SV breakpoints as single entities.

Changes to the three-dimensional genome reported in disease onset or development are often inferred from alterations in TAD boundaries ${ }^{9,28}$. For instance, CTCF activity is targeted by somatic mutations that enrich at its binding sites in colorectal, oesophageal, and liver cancers ${ }^{26,58}$. Furthermore, gains in DNA methylation at CTCF binding sites are linked to altered TAD structures in gliomas ${ }^{7}$. In primary PCa $97 \%$ of differentially methylated regions genome-wide in primary PCa are losses of DNA methylation ${ }^{59,60}$, an epigenetic process previously shown to have limited impact on CTCF chromatin binding ${ }^{61}$. This suggests that 
aberrant CTCF binding at TAD boundaries is not a hallmark of prostate oncogenesis. Our observation of stable chromatin organization supports this model. Notably, stable TAD structures observed in these primary tissues contrast previous reports of chromatin organization in cell lines derived from prostate cells ${ }^{44,62}$, highlighting the necessity of low-input protocols and primary tissues ${ }^{40}$. Our findings further support recent reports of shared higher-order chromatin organization among phenotypically distinct cell types in model organisms ${ }^{2,28,32,63-65}$. Taken together, this body of evidence suggests that large disruptions to TADs and compartments may constrain the transformation of normal to cancer cells or the divergent subtyping within prostate tumours. Instead, changes to focal chromatin interactions seem to reflect alterations in the genetic architecture leading to cancer development. Investigating these focal chromatin interactions may provide insights on the relationship between CREs, such as between enhancers and their target gene promoter ${ }^{66,67}$, to better understand the etiology of disease.

In conclusion, by bypassing technical limitations to characterize the three-dimensional genome organization across benign and tumour prostate tissue ${ }^{40}$, our work reveals the predominant stable nature of genome topology across prostate oncogenesis. Instead, alterations to discrete chromatin interactions populate the PCa genome. These impact the function of CREs, such as we report for SV-mediated CRE hijacking events. Considering the contribution of SVs across human cancers ${ }^{68}$, our collective work presents a framework inclusive of genetics, chromatin state, and three-dimensional genome organization to understand the genetic architecture across individual primary tumours. 


\section{Materials \& Methods}

\section{Patient Selection Criteria}

Patients were selected from the CPC-GENE cohort of Canadian men with indolent PCa, Gleason scores of $3+3,3+4$, and $4+3$. All primary human material was obtained with informed consent with approval of our institutional research ethics board (UHN 11-0024). The intersection of previously published data for whole genome sequencing ${ }^{33}$, RNA abundance ${ }^{41}$, and H3K27ac ChIP-seq ${ }^{42}$ led to 25 samples having data for all assays. 11 of these tested positive for ETS gene family fusions (T2E status), and 14 without. To accurately represent the presence of this subtype of $\mathrm{PCa}$ in the disease generally, and to ensure minimum read depths required to perform accurate analysis on chromatin conformation data, we selected approximately half of these remaining samples (6 T2E+ and 6 T2E-).

\section{Patient Tumour in situ low-input Hi-C Sequencing}

We followed the general in situ low input Hi-C (Low-C) protocol from Díaz et al. ${ }^{40}$, with our own re-optimization for solid tumour tissue sections. It is worth noting that throughout the protocol, the pellet would be hardly visible and would require careful pipetting. The specific modifications of the protocol are described below.

\section{Tumour Tissue Preparation}

Twelve cryopreserved-frozen PCa tumour tissue specimens were obtained from primary PCa patients as part of the Canadian PCa Genome Network (CPC-GENE) effort ${ }^{33}$. Informed consent was obtained from all patients with REB approval (UHN 11-0024). These 
tumour specimens were sectioned into $10 \mu \mathrm{m}$ sections. Sections before and after the sections used for Hi-C were stained with hematoxylin and eosin (H\&E) and assessed pathologically for $\geq 70 \%$ PCa cellularity. The percentage of infiltrating lymphocytes was also estimated by pathological assessment to be $\leq 3 \%$. Stratification into TMPRSS2-ERG (T2E)-positive or T2E-negative was determined through either whole-genome sequencing detection of the rearrangement, immunohistochemistry, or mRNA expression microarray data ${ }^{33}$.

\section{Normal Tissue Preparation}

Five snap-frozen prostate tumour-adjacent normal tissue specimens were obtained. Informed consent was obtained from all patients with REB approval (UHN 11-0024). Tissue specimens were sectioned into 5, 10, and $20 \mu \mathrm{m}$ sections. Sections used for Hi-C and RNA-seq were stained with $H \& E$ and assessed pathologically for $\geq 60 \%$ prostate glandular cellularity.

\section{Fixation and Lysis}

One or two sections (consecutive; depending on surface area) for each patient were thawed and fixed by adding $300 \mu \mathrm{L}$ of $1 \%$ formaldehyde in PBS directly onto the tissue sample, followed by a 10-minute incubation at room temperature (RT) (Supplementary Figure 1a). The formaldehyde was quenched by adding $20 \mu \mathrm{L}$ of $2.5 \mathrm{M}$ glycine to the sample reaching a final concentration of $0.2 \mathrm{M}$ followed by 5 minutes of incubation at RT. The samples were then washed three times with $500 \mu \mathrm{L}$ cold PBS and scraped off the microscope slide with a scalpel into $1.5 \mathrm{~mL}$ centrifuge tube containing $250 \mu \mathrm{L}$ of ice-cold Low-C lysis buffer (10 mM Tris-Cl pH 8.0, $10 \mathrm{mM} \mathrm{NaCl,} \mathrm{0.2 \%} \mathrm{IGEPAL} \mathrm{CA-630} \mathrm{(Sigma-Aldrich))}$ supplemented with protease inhibitor. The samples were then mixed thoroughly by gentle 
pipetting and left on ice for 20 minutes with intermittent mixing. Upon lysis, the samples were snap-frozen with liquid nitrogen and stored at $-80{ }^{\circ} \mathrm{C}$ until processing the next day. As a note, stagger fixation times when processing multiple samples to prevent needless rush and chance of under/over-fixation.

Enzyme Digestion and Overhang Fill-In

The samples stored at $-80{ }^{\circ} \mathrm{C}$ were thawed on ice and spun down at $300 \mathrm{x}$ g for 5 minutes at $4{ }^{\circ} \mathrm{C}$. The samples were then resuspended in $125 \mu \mathrm{L}$ of ice-cold $10 \mathrm{X}$ NEB2 Buffer (New England Biolabs), and again spun down at $13,000 \mathrm{xg}$ for 5 minutes at $4{ }^{\circ} \mathrm{C}$. The pellet was then resuspended in $25 \mu \mathrm{L}$ of $0.4 \%$ SDS and incubated at $65{ }^{\circ} \mathrm{C}$ for 10 minutes without agitation for permeabilization. To quench the SDS, $10 \%$ Triton X-100 in water $(12.5 \mu \mathrm{L}+75$ $\mu \mathrm{L}$ water) was then added to the samples and incubated at $37{ }^{\circ} \mathrm{C}$ for 45 minutes at $650 \mathrm{rpm}$. For enzymatic digestion, $35 \mu \mathrm{L}$ of 10X NEB2.1 buffer (New England Biolabs) was added to each sample, follow by the addition of $50 \mathrm{U}$ of $\mathrm{MboI}$ and 90 minutes incubation at $37^{\circ} \mathrm{C}$ with gentle agitation (add $30 \mathrm{U}$ first, incubate 45 minutes, followed by the addition of another 20 $\mathrm{U}$ and another 45 minutes of incubation). Upon digestion, the MboI enzyme was inactivated by incubating at $62{ }^{\circ} \mathrm{C}$ for 20 minutes. The overhangs generated by the MboI enzyme was then filled-in by adding a mix of dNTPs and DNA Polymerase I Klenow Fragment directly to each sample (10 $\mu \mathrm{L}$ of $0.4 \mathrm{mM}$ biotin-14-dCTP, $0.5 \mu \mathrm{L}$ of $10 \mathrm{mM}$ dATP, $0.5 \mu \mathrm{L}$ of $10 \mathrm{mM}$ dGTP, $0.5 \mu \mathrm{L}$ of $10 \mathrm{mM}$ dTTP, $4 \mu \mathrm{L}$ of $5 \mathrm{U} / \mu \mathrm{L}$ DNA Polymerase I Klenow Fragment). The samples were then mixed by gentle pipetting followed by incubation at $37^{\circ} \mathrm{C}$ for 90 minutes with gentle agitation.

\section{Proximity Ligation and Decrosslinking}


Upon overhang fill-in, each sample was subject to proximity ligation through the addition of $328.5 \mu \mathrm{L}$ water, $60 \mu \mathrm{L}$ of 10X T4 DNA Ligase Buffer (ThermoFisher Scientific), $50 \mu \mathrm{L}$ of $10 \%$ Triton X-100, $6 \mu \mathrm{L}$ of $20 \mathrm{mg} / \mathrm{mL}$ BSA (New England Biolabs) and $3.5 \mu \mathrm{L}$ of 5 Weiss $\mathrm{U} / \mu \mathrm{L}$ T4 DNA Ligase (ThermoFisher). The samples were mixed through gentle pipetting and incubated at RT $\left(20-22{ }^{\circ} \mathrm{C}\right)$ with rotation for 4 hours. The samples were then spun down at 13,000 x g for 5 minutes at RT and resuspended in $250 \mu \mathrm{L}$ of Extraction Buffer (50 mM Tris-Cl pH 8.0, $50 \mathrm{mM} \mathrm{NaCl}, 1 \mathrm{mM}$ EDTA, 1\% SDS) upon removal of supernatant. Next, $10 \mu \mathrm{L}$ of $20 \mathrm{mg} / \mathrm{mL}$ Proteinase K (New England Biolabs) was added to each sample and incubated at $55{ }^{\circ} \mathrm{C}$ for 30 minutes at $1,000 \mathrm{rpm}$. Then $65 \mu \mathrm{L}$ of $5 \mathrm{M} \mathrm{NaCl}$ was added to each sample and incubated at $65^{\circ} \mathrm{C}$ at $1,000 \mathrm{rpm}$ overnight.

\section{DNA Extraction}

Phenol-chloroform extraction columns were spun down at $17,000 \mathrm{xg}$ for 1 minute at $4{ }^{\circ} \mathrm{C}$ to get gel down to the bottom of the tube. The samples incubated overnight were then added to the column. Next, an equal volume $(\sim 325 \mu \mathrm{L})$ of phenol-chloroform-isoamyl alcohol mixture (25:24:1) (Sigma) was also added to the column. The column was then inverted for thorough mixing and spun down at $17,000 \mathrm{x}$ g for 5 minutes at $4{ }^{\circ} \mathrm{C}$. The surface layer on top of the gel upon spinning contains the sample and is transferred to a clean $1.5 \mathrm{~mL}$ tube $(\sim 325 \mu \mathrm{L})$. Each sample was mixed with $31.5 \mu \mathrm{L}$ of $3 \mathrm{M}$ sodium acetate, $2 \mu \mathrm{L}$ of GlycoBlue (ThermoFisher Scientific), and $504 \mu \mathrm{L}$ of $100 \%$ ethanol for DNA precipitation. The samples were inverted several times for mixing and incubated at $-80{ }^{\circ} \mathrm{C}$ for 20 minutes, followed by a centrifuge spin at $17,000 \mathrm{x}$ g for 45 minutes at $4{ }^{\circ} \mathrm{C}$. The supernatant was carefully discarded and the pellet was washed with $800 \mu \mathrm{L}$ of ice-cold $70 \%$ ethanol followed by a centrifuge spin at $17,000 \mathrm{x}$ g for 5 minutes at $4{ }^{\circ} \mathrm{C}$. The supernatant was then discarded 
and the tube was air-dried until no traces of ethanol was left prior to dissolving the DNA pellet with $30 \mu \mathrm{L}$ of Elution Buffer (Qiagen PCR Clean-Up Kit). $1 \mu \mathrm{L}$ of RNase A (ThermoFisher Scientific) was added to each sample followed by incubation at $37{ }^{\circ} \mathrm{C}$ for 15 minutes. A mix of $5 \mu \mathrm{L}$ of 10X NEB2.1 buffer (New England Biolabs), $1.25 \mu \mathrm{L}$ of $1 \mathrm{mM}$ dATP, $1.25 \mu \mathrm{L}$ of $1 \mathrm{mM}$ dCTP, $1.25 \mu \mathrm{L}$ of $1 \mathrm{mM}$ dGTP, $1 \mathrm{mM}$ of dTTP, $0.5 \mu \mathrm{L}$ of $10 \mathrm{mg} / \mathrm{mL}$ BSA, $5 \mu \mathrm{L}$ of water, $3.5 \mu \mathrm{L}$ of $3 \mathrm{U} / \mu \mathrm{L}$ T4 DNA Polymerase (New England Biolabs) was added to each sample. The samples were mixed thoroughly by gentle pipetting, and then incubated at $20^{\circ} \mathrm{C}$ for 4 hours.

\section{Fragmentation and Biotin Pull-down}

$70 \mu \mathrm{L}$ of water was added to each sample bringing total volume up to $120 \mu \mathrm{L}$, and the samples were transferred into Covaris sonication tubes. The samples were then sonicated using Covaris M220 sonicator to attain 300-700 bp fragments. For biotin pull-down using a magnetic rack, $30 \mu \mathrm{L}$ of Dynabeads MyOne Streptavidin $\mathrm{C} 1$ beads (Life Technologies) for each sample was washed once with $400 \mu \mathrm{L}$ of $1 \mathrm{X}$ B\&W buffer $+0.1 \%$ Triton $\mathrm{X}-100$. The beads were then resuspended in $120 \mu \mathrm{L}$ of $2 \mathrm{X} \mathrm{B} \& \mathrm{~W}$ buffer and transferred to the $120 \mu \mathrm{L}$ of sample (1:1 ratio). The sample was then incubated with gentle rotation at RT for 20 minutes. The supernatant was discarded and the beads were resuspended with $400 \mu \mathrm{L}$ of $1 \mathrm{X}$ B\&W buffer $+0.1 \%$ Triton $\mathrm{X}-100$ followed by a 2 -minute incubation at $55{ }^{\circ} \mathrm{C}$ with mixing. The wash was repeated once more, then resuspended in $400 \mu \mathrm{L}$ of 1X NEB2 buffer (New England Biolab). 


\section{Library Preparation and Size Selection}

The beads containing the Hi-C samples were separated on a magnetic rack to remove the supernatant. The beads were then resuspended in a total volume of $10 \mu \mathrm{L}$ for library preparation using the SMARTer ThruPLEX DNA-seq library preparation kit (Takara Biosciences) per manufacturer's protocol with an adjustment on the last step, a PCR reaction for library amplification. Upon reaching that step, the reaction was carried out on a regular PCR for two cycles to amplify the Hi-C samples off the streptavidin beads. Next, the samples were transferred onto a new tube where 20X SYBR was added. The samples were then subject to real-time qPCR and pulled out from the qPCR machine mid-exponential phase. Ultimately, this is done to reduce PCR duplication rates, a huge limitation for low-input Hi-C protocols. The Hi-C libraries were then double size-selected for 300-700 bp using Ampure XP beads and sent for BioAnalyzer analysis prior to sequencing.

\section{Hi-C Sequencing and Data Pre-processing}

Sequencing

The Hi-C libraries for each tumour sample were sent for shallow paired-end $150 \mathrm{bp}$ sequencing ( $\sim 10-15$ million reads per sample) on a NextSeq 500. Upon confirming library quality and low duplication rates $(<2 \%)$, samples were sent for deep paired-end $150 \mathrm{bp}$ sequencing with the aim of 800 million raw read pairs per sample on NovaSeq 6000 .

\section{Sequence alignment and $\mathrm{Hi}$-C artefact removal}

Paired-end FASTQ files were pre-processed with HiCUP (v0.7.2) ${ }^{69}$. Reads were truncated at MboI ligation junction sites prior to alignment with `hicup_digester`. Each mate was independently aligned to the hg38 genome and were then paired and assigned to $\mathrm{MboI}$ 
restriction sites by 'hicup_map'. 'hicup_map' uses Bowtie2 (v2.3.4) ${ }^{70}$ as the underlying aligner which has the following parameters: '--very-sensitive --no-unal --reorder'. Reads that reflect technical artefacts were filtered out with 'hicup_filter'. Duplicate reads were removed with 'hicup_deduplicator’.

Reads that came from different sequencing batches were then aggregated for each tumour sample at this stage using 'sambamba merge' (v0.6.9) ${ }^{71}$. This resulted in an average of $1.12 \times 10^{9}$ read per tumour sample (Supplementary Table 2).

\section{Contact matrix generation and balancing}

Aggregated binary alignment map (BAM) files were converted to the pairs format using pairtools (v0.2.2) $)^{72}$ and then the cooler format using the cooler package (v0.8.5) ${ }^{73}$. The pairs files were generated with the following command: 'pairtools parse -c \{genome\} --assembly hg38 -o \{output_pairs\} \{input_bam $\}^{`}$. The cooler files were generated at an initial matrix resolution of $1000 \mathrm{bp}$ with the following command: 'cooler cload pairs --assembly hg38 -c1 2 -p1 3 -c2 4 -p2 5 \{genome\}:1000 \{input_pairs\} \{output_cooler\} .

The raw contact matrices stored in the cooler file format were balanced using cooler's implementation of the ICE algorithm ${ }^{74}$ using the 'cooler balance' command. Contact matrices at different resolutions were created with the 'cooler zoomify' command.

\section{Hi-C Data Analysis}

\section{TAD identification}

Contact matrices were binned at a resolution of $40 \mathrm{kbp}$. To remove sequencing depth as a confounding factor, contact matrices for all samples were first downsampled to match the 
sequencing depth of the shallowest sample. For comparisons including cell lines, this was $120 \times 10^{6}$ contacts. For comparisons only involving primary samples, this was $300 \times 10^{6}$ contacts. This was achieved with Cooltools (v0.3.2) with the following command: 'cooltools random-sample -c 120000000 \{input\}::/resolutions/40000 \{output\}.

TADs were identified using TopDom ${ }^{75}$ on the downsampled, ICE-normalized contact matrices. To identify domains at multiple length scales, similar in concept to Artamus' gamma parameter ${ }^{76}$, TopDom was run multiple times per sample, with the window size parameter set at values between 3 and 40, inclusive (corresponding to $120 \mathrm{kbp}$ and $1.6 \mathrm{Mbp}$ ). The lower bound for the window size parameter allowed for the identification of domains multiple megabases in size at the upper end and domains $<100 \mathrm{kbp}$ at the lower end without being dominated by false calls due to sparsity of the data. Despite TopDom being more resistant to confounding by sequencing depth than other TAD calling tools ${ }^{77}$, biases in boundary persistence were evident between samples of different sequencing depth. Downsampling contact matrices to similar depths resolved these biases.

Given the stochasticity of $\mathrm{Hi}-\mathrm{C}$ sequencing, boundaries called at one window size may not correspond to the exact same location at a different window size. To attempt to resolve these different boundary calls and leverage power from multiple window sizes, boundaries for a given patient were considered at all window sizes. Boundaries within one bin $(40 \mathrm{kbp})$ of each other and called at different window sizes were marked as conflicting calls. If only two boundaries were in conflict and all the window sizes where the first boundary was called are smaller than the window sizes where the second boundary was called, the second boundary was selected since larger smoothing windows are less sensitive to small differences in contact counts. If only two boundaries were in conflict but there is no 
proper ordering of the window sizes, the boundary that was identified most often between the two was selected. If three boundaries are in conflict, the middle boundary was selected. If four or more boundaries were in conflict, the boundary that was identified most often was selected.

To determine the maximum window size for TAD calls, TAD calls were compared across window sizes for the same patient using the BPscore metric ${ }^{78}$. TAD calls are identical when the BPscore is 0 , and divergent when 1 . The cut-off window size for a single patient was determined when the difference between TAD calls at consecutive window sizes was $<$ 0.005 , twice in a row. The maximum window size was determined by the maximum window size cut-off across all samples in a comparison. For comparisons involving only primary samples, the maximum window size was determined to be $\mathrm{w}=20 \times 40 \mathrm{kbp}$. For comparisons involving cell lines, this was $\mathrm{w}=32 \times 40 \mathrm{kbp}$.

The persistence of a TAD boundary was calculated as the number of window sizes where this region was identified as a boundary.

Sample clustering by TADs

Using the TAD calls at the window size $\mathrm{w}=32 \times 40 \mathrm{kbp}$, the similarity between samples was calculated with BPscore. The resulting matrix, containing the similarity between any two samples, was used as the distance matrix for unsupervised hierarchical clustering with Ward.D2 linkage. 


\section{Compartment identification}

Contact matrices were binned at a resolution of $40 \mathrm{kbp}$, similarly to TAD identification. To remove sequencing depth as a confounding factor, contact matrices for all samples were first downsampled to match the sequencing depth of the shallowest sample. Contact matrix eigenvectors were calculated with Cooltools. To standardize the sign of each eigenvector, the $\mathrm{GC}$ content of the reference genome, binned at $40 \mathrm{kbp}$, was used as a phasing reference track. This reference track was calculated with the 'frac_gc' function from the Bioframe Python package (v0.0.12) ${ }^{79}$. The first eigenvector was used to identify compartments with the following command: 'cooltools call-compartments --bigwig --reference-track gc-content-phase.bedGraph -o \{output $\{$ input $\}$.

Identification of significant chromatin interactions

Chromatin interactions were identified in all 17 primary samples with Mustache (v1.0.2) ${ }^{80}$. Using the Cooler files from above, Mustache was run on the ICE-normalized 10 kbp contact matrix for each chromosome with the following command: 'mustache -f \{input\} -r 10000 -ch \{chromosome\} -p 8 -o \{output\}'. Interaction calls on each chromosome were merged for each sample to create a single table of interaction calls across the entire genome.

To account for variances in detection across samples and to identify similarly called interactions across samples, interaction anchors were aggregated across all samples to form a consensus set. Interaction anchors were merged if they overlapped by at least $1 \mathrm{bp}$. Interaction anchors for each sample were then mapped to the consensus set of anchors, and these new anchors were used in all subsequent analyses. 


\section{Chromatin interaction saturation analysis}

To estimate the detection of all chromatin interactions across all samples, a nonlinear regression on an asymptotic model was performed. This is similar in method to peak saturation analysis used to assess peaks detected in ChIP-seq experiments from a collection of samples ${ }^{42}$. Bootstrapping the number of unique interactions detected in a random selection of $n$ samples was calculated for $n$ ranging from 1 to 17.100 iterations of the bootstrapping process were performed. An exponential model was fit against the mean number of unique interactions detected in $n$ samples using the 'nls' and 'SSaymp' functions from the stats $\mathrm{R}$ package (v3.6.3). The model was fit to the following equation:

Where is the mean number of chromatin interactions for a given number of samples, , is the asymptotic limit of the total number of mean detected interactions, is the response for, and is the rate constant. The estimated fit was used to predict the number of samples required to reach $50 \%, 90 \%, 95 \%$, and $99 \%$ saturation of the asymptote (Supplementary Figure 3c).

\section{Structural variant breakpoint pair detection}

Breakpoint pairs for each patient were called on the merged BAM files using 'hic_breakfinder' (commit 30a0dcc6d01859797d7c263df7335fd2f52df7b8) ${ }^{27}$. Pre-calculated expected observation files for the hg38 genome were downloaded from the 'hic_breakfinder' GitHub repository on Jul 24, 2019, as per the instructions. Breakpoints were explicitly called with the following command: 'hic_breakfinder --bam-file $\{$ BAM $\}$--exp-file-inter inter_expect_1Mb.hg38.txt --exp-file-intra intra_expect_100kb.hg38.txt --name \{Sample ID $\}$ $--\min -1 \mathrm{~kb}$. 
For the T2E fusion, only one patient had the deletion identified by hic_breakfinder with default parameters (CPCG0336). Difficulties identifying SVs with hic_breakfinder have been previously noted ${ }^{47}$. After adjusting the detection threshold, we were able to identify the fusion in other samples. To ensure the T2E+ tumours were effectively stratified for future analyses, the fusion was annotated using the same coordinates for the other T2E+ samples. No other additions to breakpoint calls were made. Certain breakpoints that appeared to be artefacts were removed, as described below.

\section{Structural variant annotation and graph construction}

The contact matrix spanning $5 \mathrm{Mbp}$ upstream and downstream around the breakpoint pairs were plotted and annotated according to previously published heuristics (Supplementary Figure 4 for ${ }^{27}$ ). Breakpoint pairs that were nearby other breakpoints or did not match the heuristics in this figure were labelled as "unknown". These annotations were matched against the annotations identified from the previously published whole genome sequencing structural variants ${ }^{33}$.

Breakpoint pairs matching the following criteria were considered as detection artefacts and were ignored.

1. At least one breakpoint was $>1 \mathrm{Mbp}$

2. At least one breakpoint was surrounded by empty regions of the contact matrix

3. At least one breakpoint corresponded to a TAD or compartment boundary shared across all samples that lacked a distinct sharp edge that is indicative of a chromosomal rearrangement 
To identify unique breakpoints that were identified in multiple breakpoint pairs, breakpoints that were within $50 \mathrm{kbp}$ of each other were considered as possibly redundant calls. This distance was considered as the resolution of the non-artefactual calls is $100 \mathrm{kbp}$. Plotting the contact matrix $5 \mathrm{Mbp}$ around the breakpoint, breakpoints calls were considered the same breakpoint if the sharp edge of each breakpoint was equal to within $5 \mathrm{kbp}$.

Similar in concept to the ChainFinder algorithm ${ }^{38}$, we consider each breakpoint as a node in a graph. Nodes are connected if they are detected as a pair of breakpoints by 'hic_breakfinder'. Simple structural variants are connected components in the breakpoint graph containing only two nodes, and complex variants those with greater than two nodes. A visual representation of these graphs can be found in Supplementary Figure 4b.

\section{Determination of structural variant breakpoints altering TAD boundaries}

Patients are assigned into one of two groups using hierarchical clustering (complete linkage) with the matrix of pairwise BPscore ${ }^{78}$ values as a distance matrix. If the clustering equals the mutated samples from the non-mutated samples (i.e., the clustering matches the mutation status in this locus), then the local topology was classified as "altered" because of the SV.

Virtual $4 C$

Two parts of the $B R A F$ gene were used as anchors for virtual $4 \mathrm{C}$ data: the promoter region (1500 bp upstream, $500 \mathrm{bp}$ downstream of the TSS) and the entire gene downstream of the breakpoint. Contact frequencies from the ICE-normalized, $20 \mathrm{kbp}$ contact matrices were extracted, with the rows as the bins containing the anchor and the columns as the target regions (the $\mathrm{x}$-axes in Figure 6e). The row means were calculated to produce a single vector 
where each element is the average normalized contact frequency between the anchor of interest and the distal $20 \mathrm{kbp}$ bin. These vectors were plotted as lines in Figure 6e.

\section{Patient Tumour Tissue H3K27ac ChIP-seq}

ChIP-seq against H3K27ac was previously published for these matching samples in ${ }^{42}$. Sequencing data was processed similarly to the previous publication of this data ${ }^{42}$; however, the hg38 reference genome was used instead of hg19.

\section{Sequence alignment}

FASTQ files from single-end sequencing were aligned to the hg38 genome using Bowtie2 (v2.3.4) with the following parameters: '-x \{genome $\}-U$ input $\} 2>$ \{output_report\} $\mid$ samtools view $-\mathrm{u}>$ \{output_bam $\}$. For FASTQ files from paired-end sequencing, only the first mate was considered and reads were aligned with the following parameters: $\quad-\mathrm{x}$ \{genome $\}-\mathrm{U}\{$ input\} $-3 \quad 502>$ \{output_report\} $\mid$ samtools view $-\mathrm{u}>$ \{output_bam\}'. This ensured that all H3K27ac ChIP-seq data had the same format (single-end) and length (52 bp) before alignment to mitigate possible differences in downstream analyses due to different sequencing methods. Duplicate reads were removed with sambamba (v0.6.9) via 'sambamba markdup -r' and were then sorted by position using `sambamba sort'.

\section{Peak calling}

Peak calling was performed using MACS2 (v2.1.2) ${ }^{81}$ with the following command: 'macs 2 callpeak -g hs -f BAM -q 0.005 -B -n \{output_prefix $\}$-t \{seq_chip\} -c \{seq_input\}'. 
ENCODE hg38 blacklist regions were then removed from the narrow peaks ${ }^{82}$. Peaks calls are in Supplementary Table 8.

\section{Differential acetylation analysis}

Unique peak calls and deduplicated pull-down and control BAM files from tumour samples were loaded into $\mathrm{R}$ with the DiffBind package (v2.14.0) ${ }^{83}$ using the DESeq2 (v1.26.0) ${ }^{84}$ as the differential analysis model. 3 of the 12 samples had low quality peak calls compared to the other 9 and were not considered when calculating differential acetylation (CPCG0268, CPCG0255, and CPCG0336). We considered each unique breakpoint one at a time in the remaining 9 samples. Samples were grouped by their mutation status (i.e., a design matrix where the mutation status is the only covariate) and DiffBind's differential binding analysis method was performed to identify all differentially acetylated regions between the two groups. Acetylation peaks outside of the TAD(s) overlapping the breakpoint were filtered out. Multiple test correction with the Benjamini-Hochberg FDR method was performed on all peaks after all breakpoints were considered, due to similar group stratifications depending on the breakpoint under consideration.

\section{Structural variant breakpoint enrichment}

Structural variant breakpoint coordinates from WGS data from the CPC-GENE cohort were obtained from the International Cancer Genome Consortium (structural somatic mutations from the PRAD-CA dataset, release 28). Breakpoint coordinates were lifted over to hg38 coordinates using the liftOver function from the rtracklayer R package (v1.46.0) ${ }^{85}$. Permutation tests were performed with the regioneR R package (v1.18.0) ${ }^{86}$, selecting randomized regions from the hg38 genome, excluding the ENCODE blacklist regions ${ }^{82}$ and 
masked loci. 100 permutations were calculated and a one-sided permutation z-test was used to calculate statistical significance.

\section{Primary Tissue RNA Data Analysis}

Tumour sample RNA sequencing

Total RNA was extracted for the CPC-GENE tumour samples as previously described

41. Briefly, total RNA was extracted with mirVana miRNA Isolation Kit (Life Technologies) according to the manufacturer's instructions. RNA samples were sent to BGI Americas where it underwent QC and DNase treatment. For each sample, $200 \mathrm{ng}$ of total RNA was used to construct a TruSeq strand-specific library with the Ribo Zero protocol (Illumina, Cat. \#RS-122-2203). The libraries were sequenced on a HiSeq 2000 to a minimal target of 180 million, 2 x 100 bp paired-end reads.

RNA sequencing data pre-processing

RNA sequencing FASTQ files were pseudo-aligned to the hg38 genome using Kallisto (v0.46.1) ${ }^{87}$ with the following command: 'kallisto quant --bootstrap-samples 100 --pseudobam --threads $8 \quad$--index $\quad /$ path/to/GRCh38.idx $\quad$--output-dir \{output_dir\} \{input_R1.fastq.gz\} \{input_R2.fastq.gz\} ${ }^{\prime}$.

\section{Differential gene expression analysis}

To assess whether SVs were associated with local gene expression changes, we considered each unique breakpoint one at a time. For each breakpoint, we compared the gene expression between the mutated and non-mutated tumour samples using Sleuth (v0.30.0) ${ }^{88,89}$ with a linear model where the mutation status was the only covariate. To reduce the chance of 
falsely identifying genes as differentially expressed, only genes located within the TADs (window size 20) containing breakpoints were considered. Fold-change estimates of each transcript were assessed for significance using a Wald test. Transcript-level p-values are combined to create gene-level p-values using the Lancaster aggregation method provided by the Sleuth package. Correcting for multiple tests was then performed with the Benjamini-Hochberg FDR correction for all genes that were potentially altered in the mutated sample(s).

\section{Data Availability}

Whole genome and RNA sequencing data is available in the European Genome-Phenome Archive (EGA) under accession number EGAS00001000900. H3K27ac ChIP sequencing data is available in EGA under accession number EGAS00001002496. Hi-C sequencing data is under submission to the European Genome-Phenome Archive (EGAS00001005014). TADs and chromatin interactions are available in the Gene Expression Omnibus (GEO) archive under accession number GSE164347. Hi-C sequencing data from cell lines was obtained from GEO under expression number GSE118629 (22Rv1, RWPE1, and $\mathrm{C} 4-2 \mathrm{~B}$ cell lines) and from the 4D Nucleome under accession numbers 4DNFI6HDY7WZ (H1-hESC Rep 1), 4DNFITH978XV (H1-hESC Rep 2), 4DNFIT64Q7A3 (HAP-1 Rep 1), 4DNFINSKEZND (HAP-1 Rep 2), 4DNFIIV4M7TF (GM12878 Rep 1), and 4DNFIXVAKX9Q (GM12878 Rep 2). Processed data is available on CodeOcean (https://codeocean.com/capsule/5232537/tree). 


\section{Code Availability}

Code for data processing, analysis, and plotting can be found on CodeOcean (https://codeocean.com/capsule/5232537/tree).

\section{Disclosure of Potential Conflicts of Interest}

No potential conflicts of interest were disclosed.

\section{Author Contributions}

S.Z., J.R.H., and M.L. conceptualized the study. S.Z. designed and conducted all the experiments with help from C.A. G.G., AND K.K. J.R.H. implemented all the computational and statistical approaches and analyses. R.H.-W. pre-processed the RNA-seq data from the primary tumours. Figures were designed by S.Z. and J.R.H. The manuscript was written by S.Z., J.H., and M.L with assistance from all authors. T.v.d.K., M.F., P.C.B., R.G.B., and M.L. supervised the study. M.L. oversaw the study.

\section{Acknowledgements}

We thank all the Lupien lab members for their feedback, as well as Jesse Dixon for his support with hic_breakfinder and interpretation of structural variant calls. This work is supported by Prostate Cancer Foundation Canada, Ontario Institute for Cancer Research funded by the Government of Ontario, the Princess Margaret Cancer Foundation (R.G.B. and M.L.), Princess Margaret Cancer Centre Department of Surgical Oncology (M.F.), Princess 
Margaret Cancer Centre Genetics and Epigenetic Program (M.F. and M.L.), University of Toronto Department of Surgery Division of Urology (M.F.), Movember Foundation (RS2014-04 to M.L. and RS2014-01 to P.C.B.), the Radiation Medicine Program Academic Enrichment Fund (R.G.B.), Terry Fox Research Institute New Investigator Award (P.C.B.), Canadian Institute of Health Research (CIHR; FRN-153234 to M.L.) and New Investigator Award (P.C.B. and M.L.), Canadian Cancer Society Research Scientist Award (R.G.B.), Cancer Society Impact Award (P.C.B), Investigator Award from the Ontario Institute for Cancer Research (M.L. and P.C.B), and Movember Rising Star Award from PCa Canada (M.L. and P.C.B). 


\section{References}

1. Finn, E. H. \& Misteli, T. Molecular basis and biological function of variability in spatial genome organization. Science 365, eaaw9498 (2019).

2. Dixon, J. R. et al. Topological domains in mammalian genomes identified by analysis of chromatin interactions. Nature 485, 376-380 (2012).

3. Nora, E. P. et al. Spatial partitioning of the regulatory landscape of the X-inactivation centre. Nature 485, 381-385 (2012).

4. Pombo, A. \& Dillon, N. Three-dimensional genome architecture: players and mechanisms. Nat. Rev. Mol. Cell Biol. 16, 245-257 (2015).

5. Northcott, P. A. et al. Enhancer hijacking activates GFI1 family oncogenes in medulloblastoma. Nature 511, 428-434 (2014).

6. Gröschel, S. et al. A single oncogenic enhancer rearrangement causes concomitant EVI1 and GATA2 deregulation in leukemia. Cell 157, 369-381 (2014).

7. Flavahan, W. A. et al. Insulator dysfunction and oncogene activation in IDH mutant gliomas. Nature 529, 110-114 (2016).

8. Haller, F. et al. Enhancer hijacking activates oncogenic transcription factor NR4A3 in acinic cell carcinomas of the salivary glands. Nat. Commun. 10, 368 (2019).

9. Oudelaar, A. M. \& Higgs, D. R. The relationship between genome structure and function. Nat. Rev. Genet. 571, 489 (2020).

10. Despang, A. et al. Functional dissection of the Sox9-Kcnj2 locus identifies nonessential and instructive roles of TAD architecture. Nat. Genet. 51, 1263-1271 (2019).

11. Williamson, I. et al. Developmentally regulated expression is robust to TAD perturbations. Development 146, (2019).

12. Zhou, S., Treloar, A. E. \& Lupien, M. Emergence of the Noncoding Cancer Genome: A Target of 
Genetic and Epigenetic Alterations. Cancer Discov. 6, 1215-1229 (2016).

13. Rheinbay, E. et al. Analyses of non-coding somatic drivers in 2,658 cancer whole genomes. Nature 578, 102-111 (2020).

14. Li, Y. et al. Patterns of somatic structural variation in human cancer genomes. Nature 578, $112-121(2020)$.

15. Vinagre, J. et al. Frequency of TERT promoter mutations in human cancers. Nat. Commun. 4, $2185(2013)$.

16. Huang, F. W. et al. Highly Recurrent TERT Promoter Mutations in Human Melanoma. Science 339, 957-959 (2013).

17. Stern, J. L., Theodorescu, D., Vogelstein, B., Papadopoulos, N. \& Cech, T. R. Mutation of the TERT promoter, switch to active chromatin, and monoallelic TERT expression in multiple cancers. Genes Dev. 29, 2219-2224 (2015).

18. Bailey, S. D. et al. Noncoding somatic and inherited single-nucleotide variants converge to promote ESR1 expression in breast cancer. Nat. Genet. 48, 1260-1266 (2016).

19. Zhou, S. et al. Noncoding mutations target cis-regulatory elements of the FOXA1 plexus in prostate cancer. Nat. Commun. 11, 441 (2020).

20. Parolia, A. et al. Distinct structural classes of activating FOXA1 alterations in advanced prostate cancer. Nature vol. 571 413-418 (2019).

21. Jeselsohn, R., Buchwalter, G., De Angelis, C., Brown, M. \& Schiff, R. ESR1 mutations-a mechanism for acquired endocrine resistance in breast cancer. Nat. Rev. Clin. Oncol. 12, 573-583 (2015).

22. Robinson, J. \& Carroll, J. FoxA1 is a Key Mediator of Hormonal Response in Breast and Prostate Cancer. Front. Endocrinol. 3, (2012).

23. Fu, X. et al. FOXA1 overexpression mediates endocrine resistance by altering the ER transcriptome and IL-8 expression in ER-positive breast cancer. Proc. Natl. Acad. Sci. U. S. A. 113, E6600-E6609 (2016). 
24. $\mathrm{Fu}$, X. et al. FOXA1 upregulation promotes enhancer and transcriptional reprogramming in endocrine-resistant breast cancer. Proc. Natl. Acad. Sci. U. S. A. (2019) doi:10.1073/pnas.1911584116.

25. Maurano, M. T. et al. Large-scale identification of sequence variants influencing human transcription factor occupancy in vivo. Nat. Genet. 47, 1393-1401 (2015).

26. Guo, Y. A. et al. Mutation hotspots at CTCF binding sites coupled to chromosomal instability in gastrointestinal cancers. Nat. Commun. 9, 1-14 (2018).

27. Dixon, J. R. et al. Integrative detection and analysis of structural variation in cancer genomes. Nat. Genet. 50, 1388-1398 (2018).

28. Akdemir, K. C. et al. Disruption of chromatin folding domains by somatic genomic rearrangements in human cancer. Nat. Genet. (2020) doi:10.1038/s41588-019-0564-y.

29. Hnisz, D. et al. Activation of proto-oncogenes by disruption of chromosome neighborhoods. Science 351, 1454-1458 (2016).

30. Lupiáñez, D. G. et al. Disruptions of topological chromatin domains cause pathogenic rewiring of gene-enhancer interactions. Cell 161, 1012-1025 (2015).

31. Allou, L. et al. Non-coding deletions identify Maenli lncRNA as a limb-specific En1 regulator. Nature 592, 93-98 (2021).

32. Iyyanki, T. et al. Subtype-associated epigenomic landscape and 3D genome structure in bladder cancer. Genome Biol. 22, 105 (2021).

33. Fraser, M. et al. Genomic hallmarks of localized, non-indolent prostate cancer. Nature 541, 359-364 (2017).

34. Takeda, D. Y. et al. A Somatically Acquired Enhancer of the Androgen Receptor Is a Noncoding Driver in Advanced Prostate Cancer. Cell doi:10.1016/j.cell.2018.05.037.

35. Rosen, P. et al. Clinical potential of the ERG oncoprotein in prostate cancer. Nat. Rev. Urol. 9 , 131-137 (2012).

36. Quigley, D. A. et al. Genomic Hallmarks and Structural Variation in Metastatic Prostate Cancer. 
Cell 174, 758-769.e9 (2018).

37. Cancer Genome Atlas Research Network. The Molecular Taxonomy of Primary Prostate Cancer. Cell 163, 1011-1025 (2015).

38. Baca, S. C. et al. Punctuated evolution of prostate cancer genomes. Cell 153, 666-677 (2013).

39. Lieberman-Aiden, E. et al. Comprehensive mapping of long-range interactions reveals folding principles of the human genome. Science 326, 289-293 (2009).

40. Díaz, N. et al. Chromatin conformation analysis of primary patient tissue using a low input Hi-C method. Nat. Commun. 9, 4938 (2018).

41. Chen, S. et al. Widespread and Functional RNA Circularization in Localized Prostate Cancer. Cell 176, 831-843.e22 (2019).

42. Kron, K. J. et al. TMPRSS2-ERG fusion co-opts master transcription factors and activates NOTCH signaling in primary prostate cancer. Nat. Genet. (2017) doi:10.1038/ng.3930.

43. Mazrooei, P. et al. Cistrome Partitioning Reveals Convergence of Somatic Mutations and Risk Variants on Master Transcription Regulators in Primary Prostate Tumors. Cancer Cell 36, 674-689.e6 (2019).

44. Rhie, S. K. et al. A high-resolution 3D epigenomic map reveals insights into the creation of the prostate cancer transcriptome. Nat. Commun. 10, 4154 (2019).

45. Takayama, N. et al. The Transition from Quiescent to Activated States in Human Hematopoietic Stem Cells Is Governed by Dynamic 3D Genome Reorganization. Cell Stem Cell 28, 488-501.e10 (2021).

46. Johnstone, S. E. et al. Large-Scale Topological Changes Restrain Malignant Progression in Colorectal Cancer. Cell 182, 1474-1489.e23 (2020).

47. Ho, S. S., Urban, A. E. \& Mills, R. E. Structural variation in the sequencing era. Nat. Rev. Genet. 21, 171-189 (2020).

48. Berger, M. F. et al. The genomic complexity of primary human prostate cancer. Nature $\mathbf{4 7 0}$, 214-220 (2011). 
49. Mazrooei, P. et al. Somatic Mutations and Risk-Variants Converge on Cis-Regulatory Elements to Reveal the Cancer Driver Transcription Regulators in Primary Prostate Tumors. Cancer cell 36, 674-689 (2019).

50. Gasperini, M. et al. A Genome-wide Framework for Mapping Gene Regulation via Cellular Genetic Screens. Cell 176, 377-390.e19 (2019).

51. Tomlins, S. A. et al. Recurrent fusion of TMPRSS2 and ETS transcription factor genes in prostate cancer. Science 310, 644-648 (2005).

52. Tomlins, S. A. et al. Distinct classes of chromosomal rearrangements create oncogenic ETS gene fusions in prostate cancer. Nature 448, 595-599 (2007).

53. Viswanathan, S. R. et al. Structural Alterations Driving Castration-Resistant Prostate Cancer Revealed by Linked-Read Genome Sequencing. Cell (2018) doi:10.1016/j.cell.2018.05.036.

54. Wu, S. et al. Circular ecDNA promotes accessible chromatin and high oncogene expression. Nature 575, 699-703 (2019).

55. Kumar, P. et al. ATAC-seq identifies thousands of extrachromosomal circular DNA in cancer and cell lines. Science Advances 6, eaba2489 (2020).

56. Morton, A. R. et al. Functional Enhancers Shape Extrachromosomal Oncogene Amplifications. Cell 179, 1330-1341.e13 (2019).

57. Shoshani, O. et al. Chromothripsis drives the evolution of gene amplification in cancer. Nature 591, 137-141 (2021).

58. Katainen, R. et al. CTCF/cohesin-binding sites are frequently mutated in cancer. Nat. Genet. 47, 818-821 (2015).

59. Zhao, S. G. et al. The DNA methylation landscape of advanced prostate cancer. Nat. Genet. 52, $778-789$ (2020).

60. Yu, Y. P. et al. Whole-genome methylation sequencing reveals distinct impact of differential methylations on gene transcription in prostate cancer. Am. J. Pathol. 183, 1960-1970 (2013).

61. Role of DNA Methylation in Modulating Transcription Factor Occupancy. Cell Rep. 12, 
1184-1195 (2015).

62. Taberlay, P. C. et al. Three-dimensional disorganization of the cancer genome occurs coincident with long-range genetic and epigenetic alterations. Genome Res. 26, 719-731 (2016).

63. Rao, S. S. P. et al. A 3D map of the human genome at kilobase resolution reveals principles of chromatin looping. Cell 159, 1665-1680 (2014).

64. Ing-Simmons, E. et al. Independence of chromatin conformation and gene regulation during Drosophila dorsoventral patterning. Nat. Genet. 53, 487-499 (2021).

65. Ghavi-Helm, Y. et al. Highly rearranged chromosomes reveal uncoupling between genome topology and gene expression. Nat. Genet. 51, 1272-1282 (2019).

66. Gasperini, M., Tome, J. M. \& Shendure, J. Towards a comprehensive catalogue of validated and target-linked human enhancers. Nat. Rev. Genet. 21, 292-310 (2020).

67. Nasser, J. et al. Genome-wide enhancer maps link risk variants to disease genes. Nature (2021) doi:10.1038/s41586-021-03446-x.

68. Hanahan, D. \& Weinberg, R. A. Hallmarks of cancer: the next generation. Cell 144, 646-674 (2011).

69. Wingett, S. W. et al. HiCUP: pipeline for mapping and processing Hi-C data. F1000Research vol. $41310(2015)$.

70. Langmead, B. \& Salzberg, S. L. Fast gapped-read alignment with Bowtie 2. Nat. Methods 9 , $357-359$ (2012).

71. Tarasov, A., Vilella, A. J., Cuppen, E., Nijman, I. J. \& Prins, P. Sambamba: fast processing of NGS alignment formats. Bioinformatics 31, 2032-2034 (2015).

72. Goloborodko, A., Abdennur, N., Venev, S., hbbrandao \& gfudenberg. mirnylab/pairtools: v0.2.2. (Zenodo, 2019). doi:10.5281/ZENODO.2533337.

73. Abdennur, N. et al. mirnylab/cooler: v0.8.5. (Zenodo, 2019). doi:10.5281/ZENODO.2633272.

74. Imakaev, M. et al. Iterative correction of Hi-C data reveals hallmarks of chromosome organization. Nat. Methods 9, 999-1003 (2012). 
75. Shin, H. et al. TopDom: an efficient and deterministic method for identifying topological domains in genomes. Nucleic Acids Res. 44, e70 (2016).

76. Filippova, D., Patro, R., Duggal, G. \& Kingsford, C. Identification of alternative topological domains in chromatin. Algorithms Mol. Biol. 9, 14 (2014).

77. Dali, R. \& Blanchette, M. A critical assessment of topologically associating domain prediction tools. Nucleic Acids Res. 45, 2994-3005 (2017).

78. Zaborowski, R. \& Wilczyński, B. BPscore: An Effective Metric for Meaningful Comparisons of Structural Chromosome Segmentations. J. Comput. Biol. 26, 305-314 (2019).

79. Abdennur, N., Goloborodko, A., Abraham, S. \& aafkevandenberg. mirnylab/bioframe: v0.0.12-doi. (Zenodo, 2020). doi:10.5281/ZENODO.3897574.

80. ay-lab, Gezer, T. \& Ardakany, A. R. ay-lab/mustache v1.0.1. (Zenodo, 2020). doi:10.5281/ZENODO.4008045.

81. Zhang, Y. et al. Model-based analysis of ChIP-Seq (MACS). Genome Biol. 9, R137 (2008).

82. Amemiya, H. M., Kundaje, A. \& Boyle, A. P. The ENCODE Blacklist: Identification of Problematic Regions of the Genome. Sci. Rep. 9, 9354 (2019).

83. DiffBind. http://bioconductor.org/packages/DiffBind/.

84. Love, M. I., Huber, W. \& Anders, S. Moderated estimation of fold change and dispersion for RNA-seq data with DESeq2. Genome Biol. 15, 550 (2014).

85. Lawrence, M., Gentleman, R. \& Carey, V. rtracklayer: an R package for interfacing with genome browsers. Bioinformatics 25, 1841-1842 (2009).

86. Gel, B. et al. regioneR: an R/Bioconductor package for the association analysis of genomic regions based on permutation tests. Bioinformatics 32, 289-291 (2016).

87. Bray, N. L., Pimentel, H., Melsted, P. \& Pachter, L. Near-optimal probabilistic RNA-seq quantification. Nat. Biotechnol. 34, 525-527 (2016).

88. Pimentel, H., Bray, N. L., Puente, S., Melsted, P. \& Pachter, L. Differential analysis of RNA-seq incorporating quantification uncertainty. Nat. Methods 14, 687-690 (2017). 
89. Yi, L., Pimentel, H., Bray, N. L. \& Pachter, L. Gene-level differential analysis at transcript-level resolution. Genome Biol. 19, 53 (2018).

90. Kamada, T. \& Kawai, S. An algorithm for drawing general undirected graphs. Information Processing Letters 31, 7-15 (1989).

91. Hagberg, A. A., Schult, D. A. \& Swart, P. J. Exploring Network Structure, Dynamics, and Function using NetworkX. in Proceedings of the Python in Science Conference (SciPy) (eds. Varoquaux, G., Vaught, T. \& Millman, J.) 11-15 (2008). 
bioRxiv preprint doi: https://doi.org/10.1101/2021.01.05.425333; this version posted April 27, 2021. The copyright holder for this preprint (which was not certified by peer review) is the author/funder, who has granted bioRxiv a license to display the preprint in perpetuity. It is made available under aCC-BY-NC 4.0 International license.

\section{Figures}

\section{Figure 1}

a

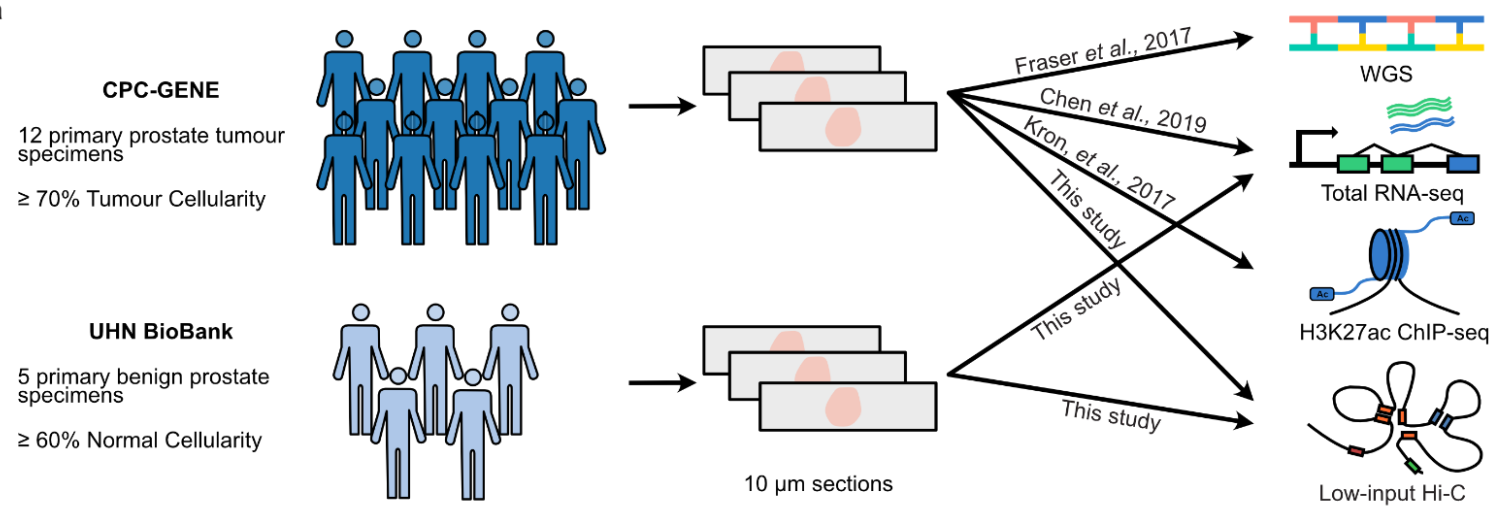

b

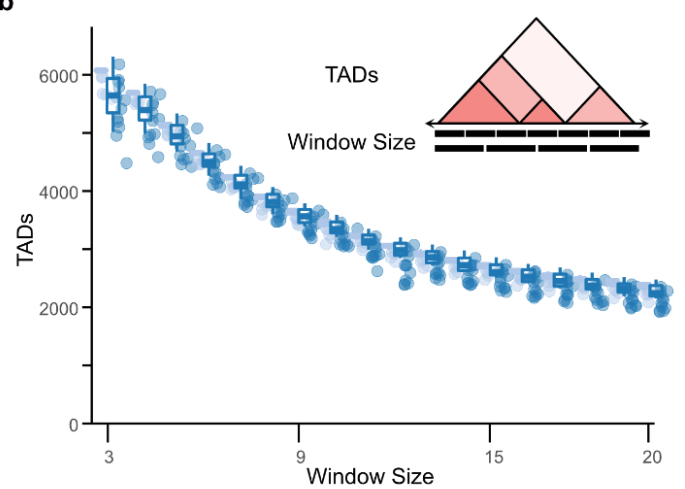

c

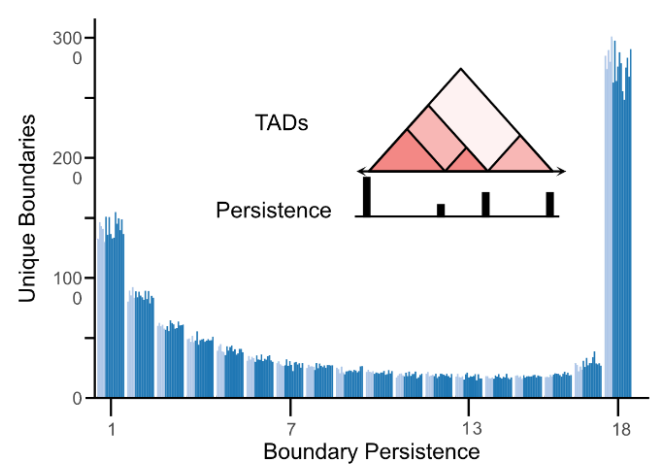

d

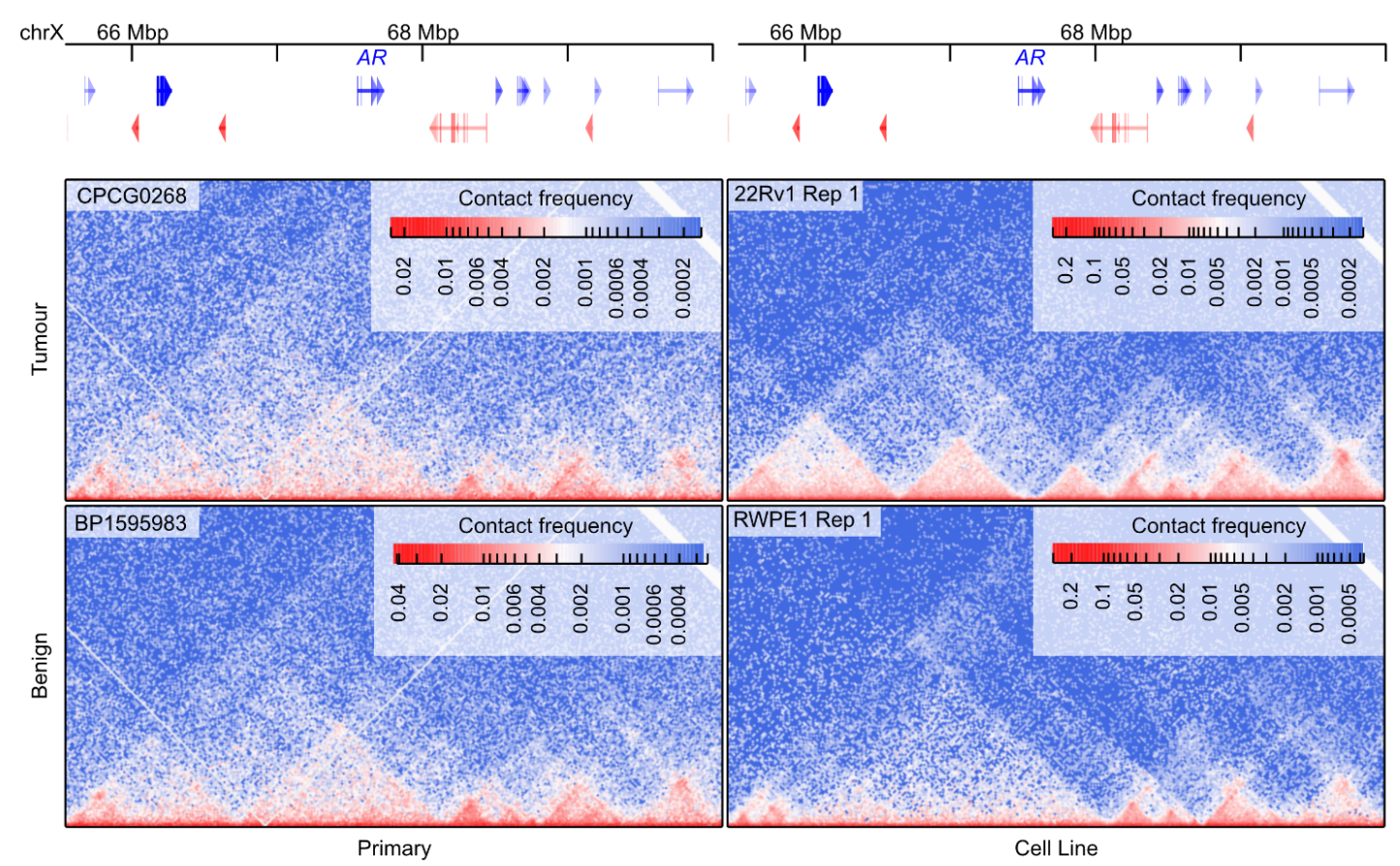


Topologically associated domains are stable over prostate oncogenesis. a. The sample collection and data usage of primary prostate samples in this study. $10 \mu \mathrm{m}$ sections from 6 tumours previously identified as $\mathrm{T} 2 \mathrm{E}+$ and 6 T2E- were used for $\mathrm{Hi}-\mathrm{C}$ sequencing. 5 additional $10 \mu \mathrm{m}$ sections were collected from benign prostate specimens in the UHN BioBank. b-c. A comparison of the number of TADs detected at multiple window sizes (b) and boundary persistence (c) in each patient sample, with inset schematics. d. Contact matrices around the $A R$ gene demonstrate a difference in chromatin organization between primary samples and cell lines. Hi-C data for 22Rv1 and RWPE1 cell lines obtained from ${ }^{44}$. 


\section{Figure 2}

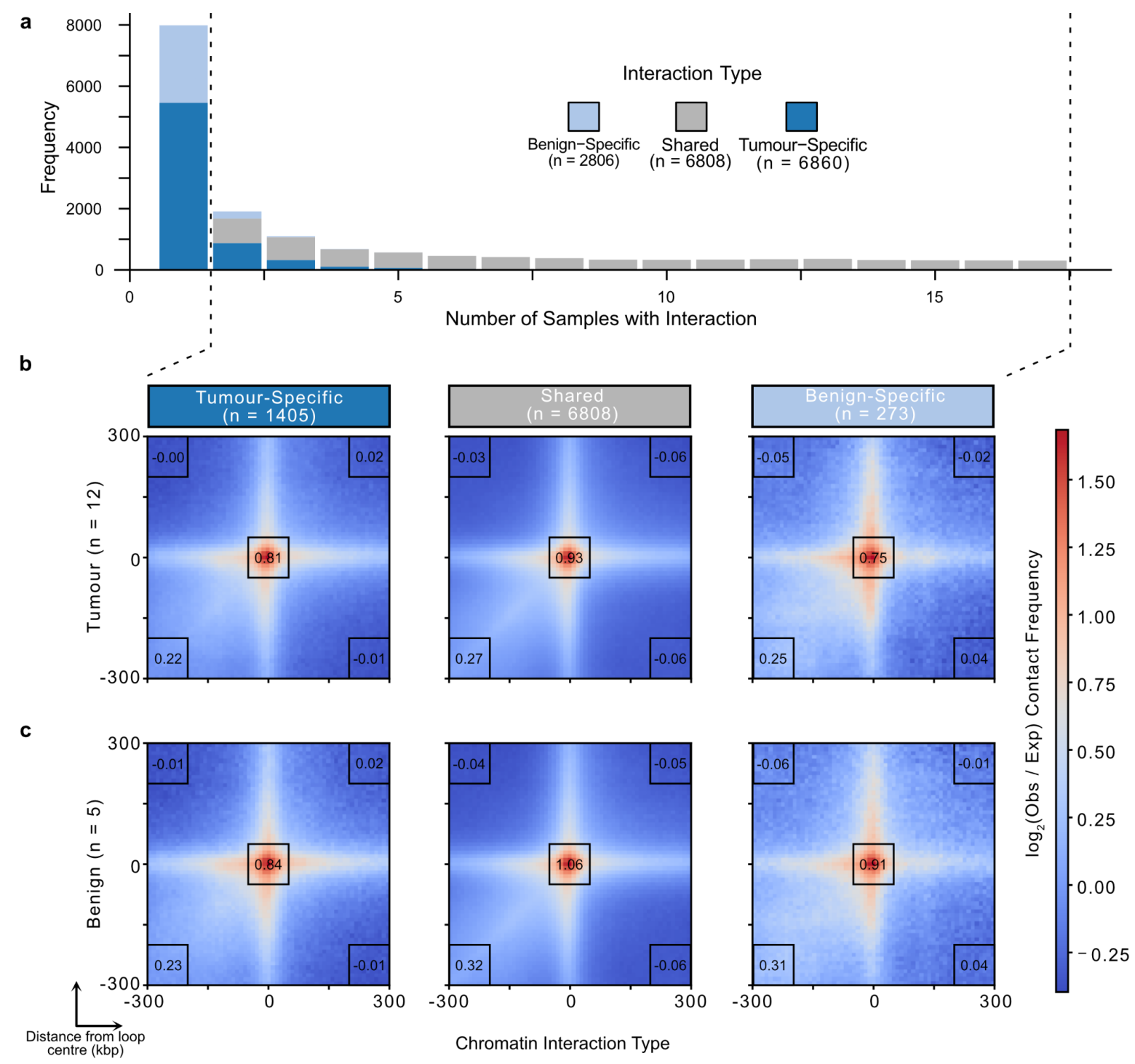

Focal chromatin interactions display subtle differences between benign and tumour

tissue. a. Stacked bar plots of the number of samples chromatin interactions were identified in. b-c. Aggregate peak analysis of tumour (b) or benign (c) contacts in tumour-specific, benign-specific, and shared interactions identified in two or more samples. Regions plotted are $+/-300 \mathrm{kbp}$ around the centre of each identified interaction. Inset numbers are the mean $\log 2(\mathrm{obs} / \mathrm{exp}$ ) contact frequencies within the $100 \mathrm{kbp}$ x $100 \mathrm{kbp}$ black boxes. 


\section{Figure 3}

a

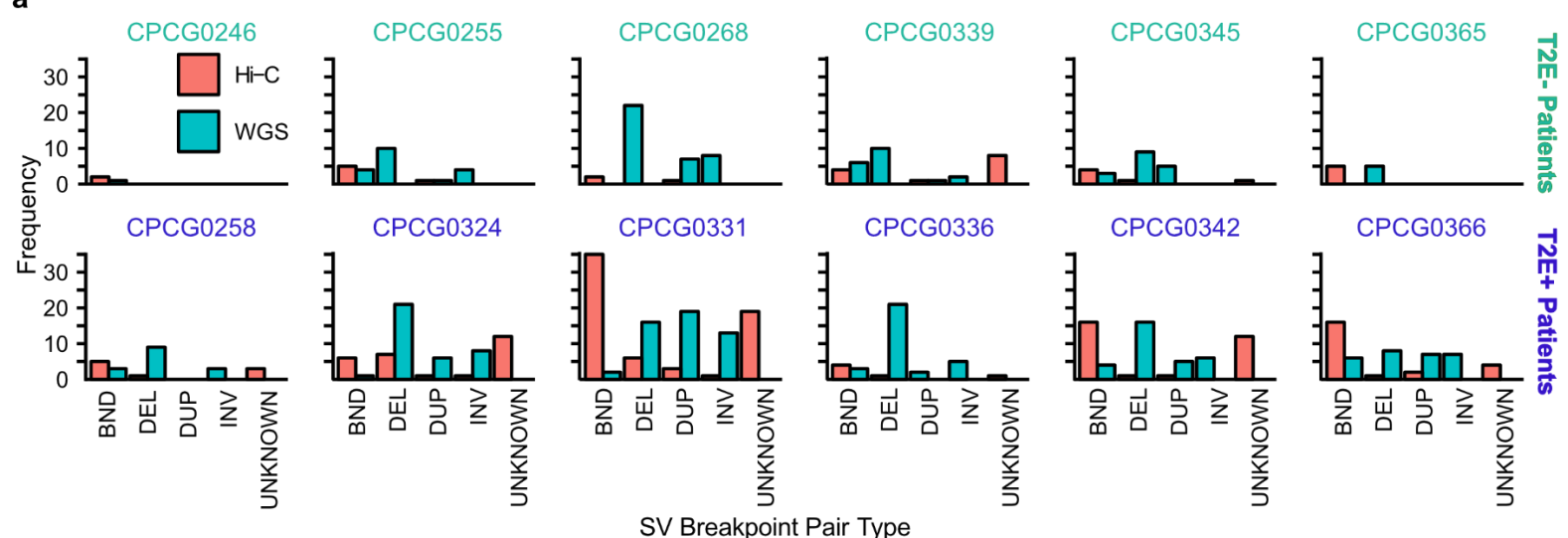

b
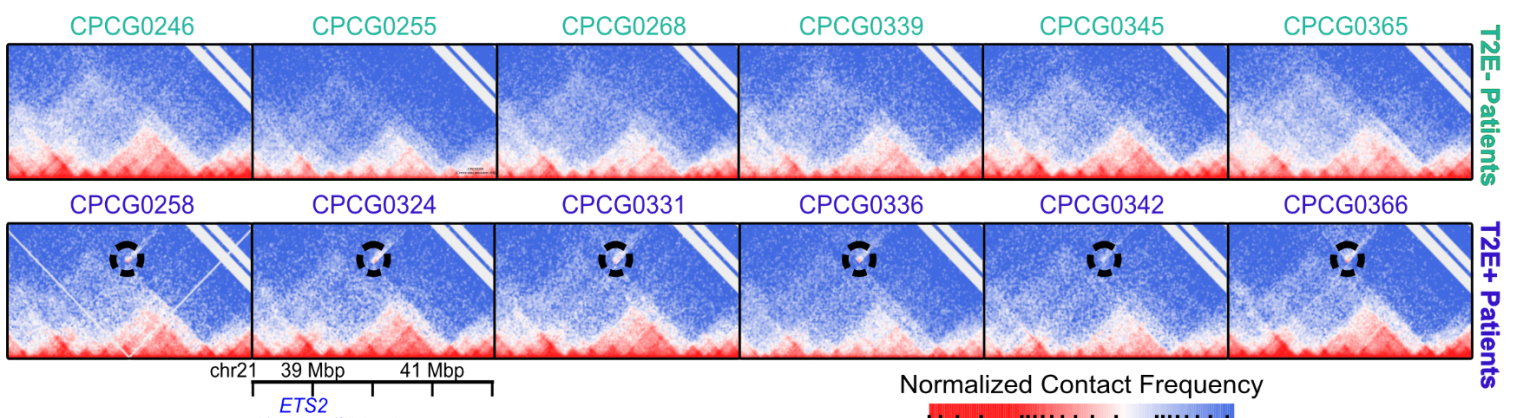

C

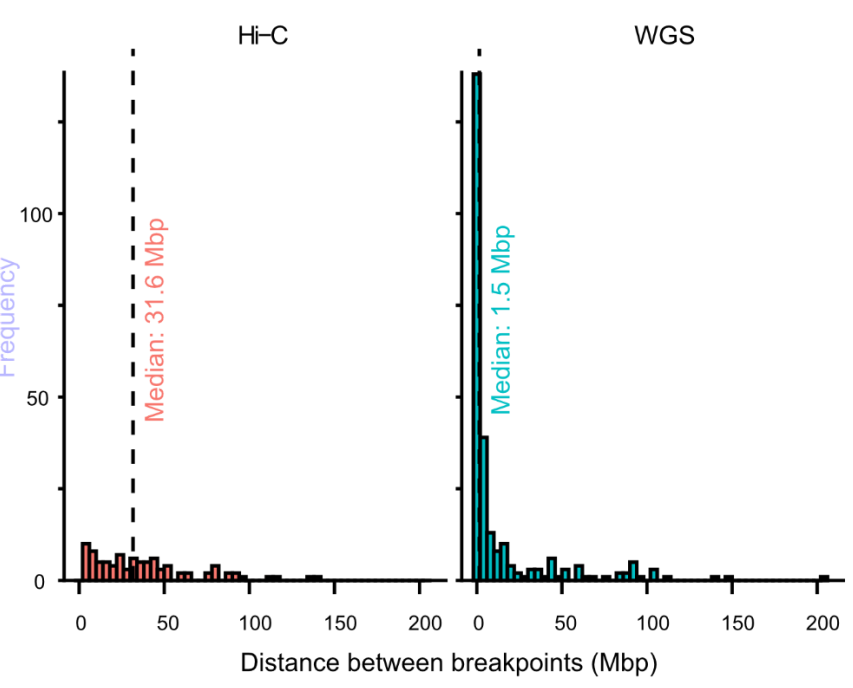

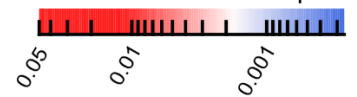

d

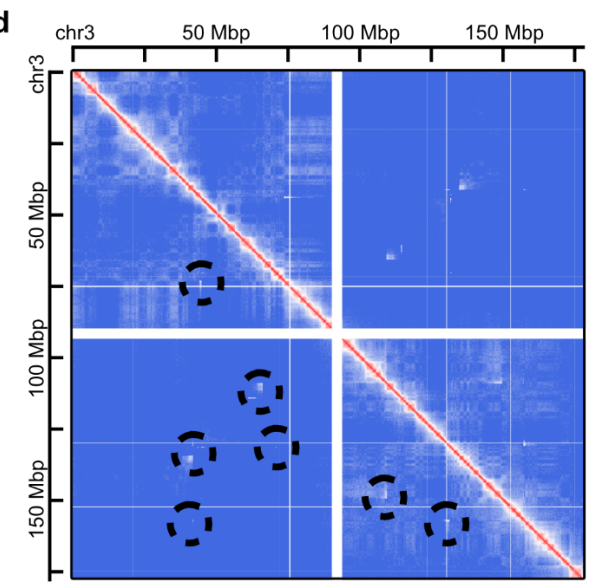

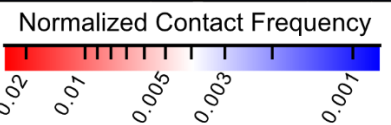

SVs are identified in primary tissue through chromatin conformation capture. a. Barplot of SV breakpoint pairs identified by $\mathrm{Hi}-\mathrm{C}$ and $\mathrm{WGS}^{33}$ on matched samples. BND = inter-chromosomal translocation, DEL = deletion, DUP $=$ duplication, INV = inversion, UNKNOWN = breakpoint pair of unknown type. b. Hi-C contact matrices of the chr21:37-42 
Mbp locus harbouring the TMPRSS2 and ERG genes. Circles indicate increased contact between TMPRSS2 and ERG in the T2E+ tumours. c. Histogram showing the distance between breakpoints on the same chromosome detected by Hi-C (left) versus WGS ${ }^{33}$ (right). d. An example of a complex set of rearrangement spanning both arms of chromosome 3 in a patient. 


\section{Figure 4}

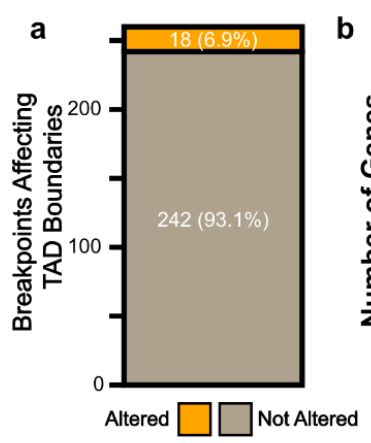

c
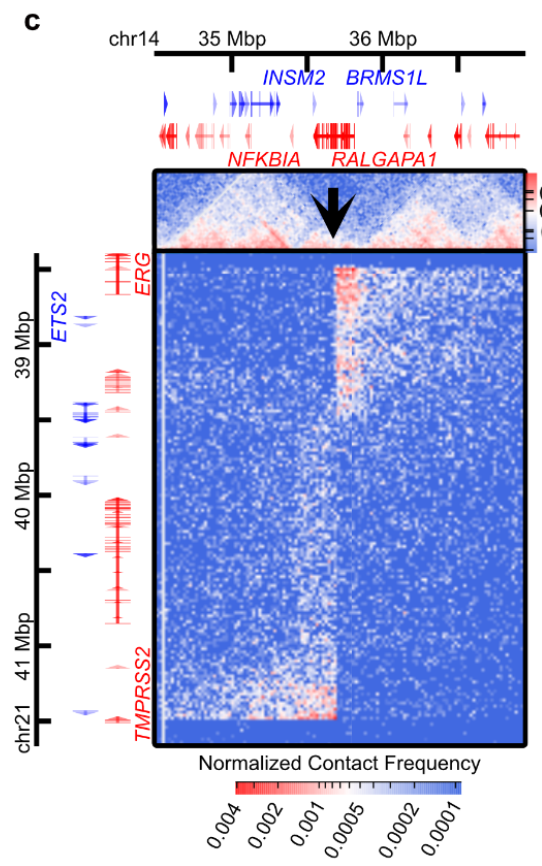

g

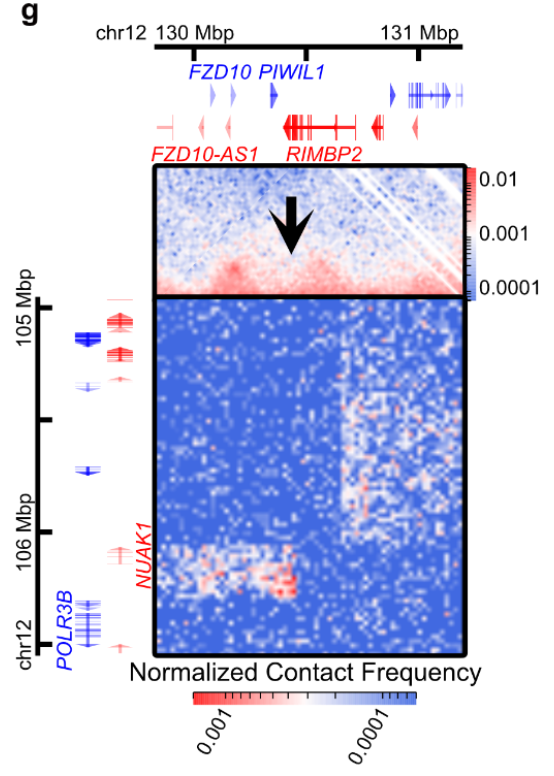

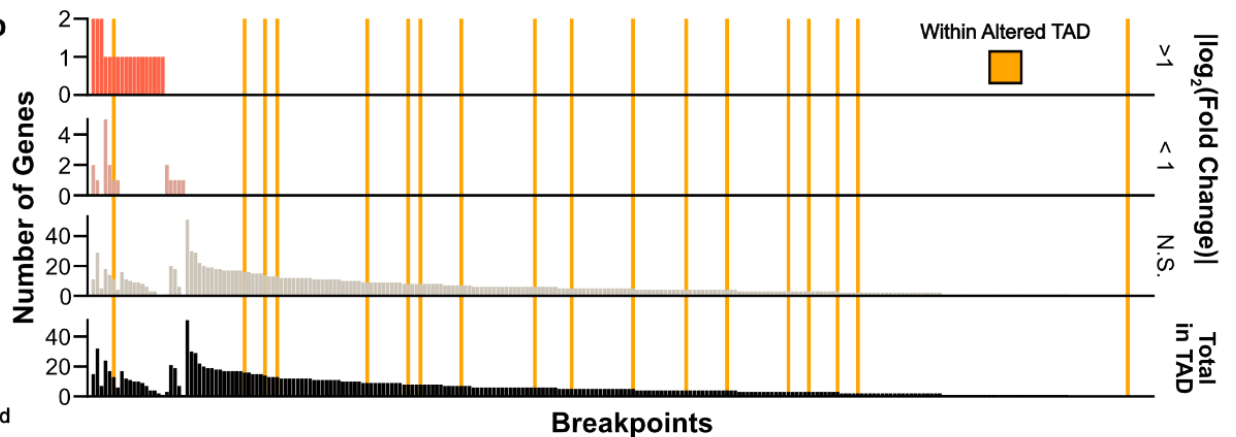

d

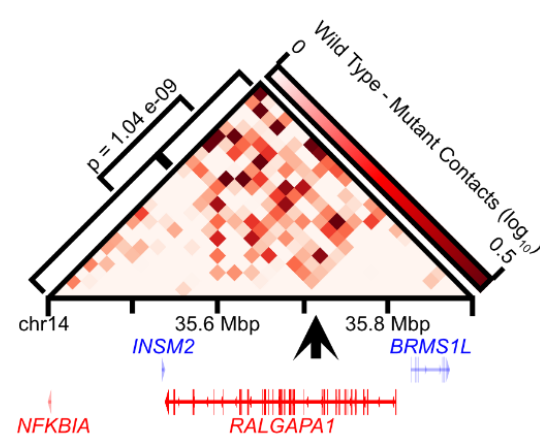

f
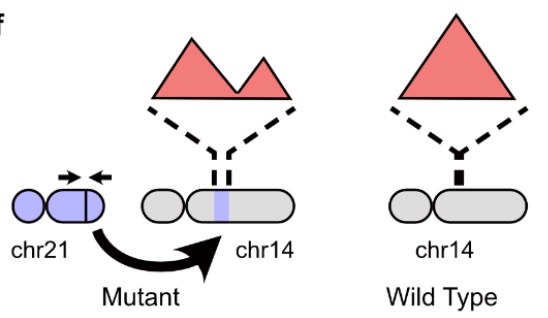

chr14

Wild Type

h

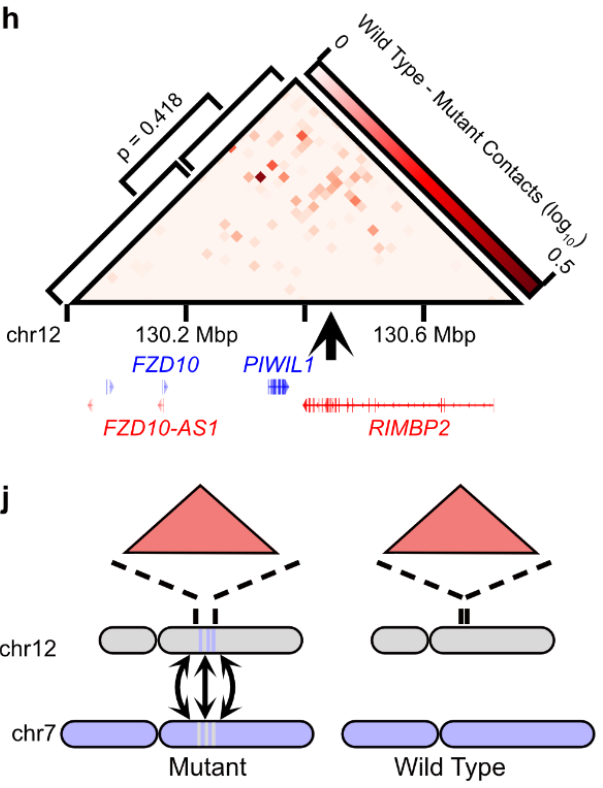

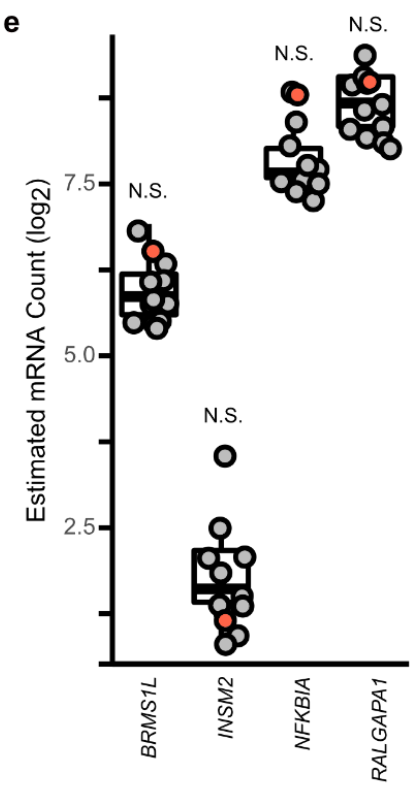

Mutated

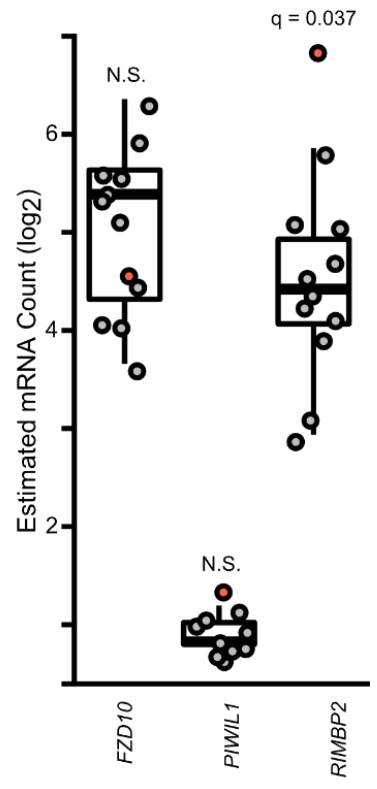


SVs can alter TADs or gene expression around breakpoints, but rarely alters both. a. A count of the number of SV breakpoints associated with altered TAD boundaries. b. Bar plot showing the number of genes differentially expressed around SV breakpoints. c-f. An example of an SV that alters TAD boundaries without significantly affecting gene expression of the nearby genes. c. The contact matrix showing a translocation of the TMPRSS2-ERG locus into chr14 in the RALGAPAl gene. d. The differential contact matrix between the tumour containing this translocation and another tumour without it to show the decreased contacts between sites upstream and downstream of the insertion site. e. Expression of the genes within the broken TAD show no significant changes to their expression. f. A schematic representation of the translocation. g-j. An example of an SV that does not alter TAD boundaries but does alter the expression of a nearby gene. g. The contact matrix showing a complex rearrangement around the RIMBP2 gene. h. The differential contact matrix between the tumour containing this translocation and another tumour without it to show the decreased contacts between sites upstream and downstream of the insertion site. i. Expression of the genes within the broken TAD show no significant changes to their expression. $\mathbf{j}$. A schematic representation of the translocation. 


\section{Figure 5}
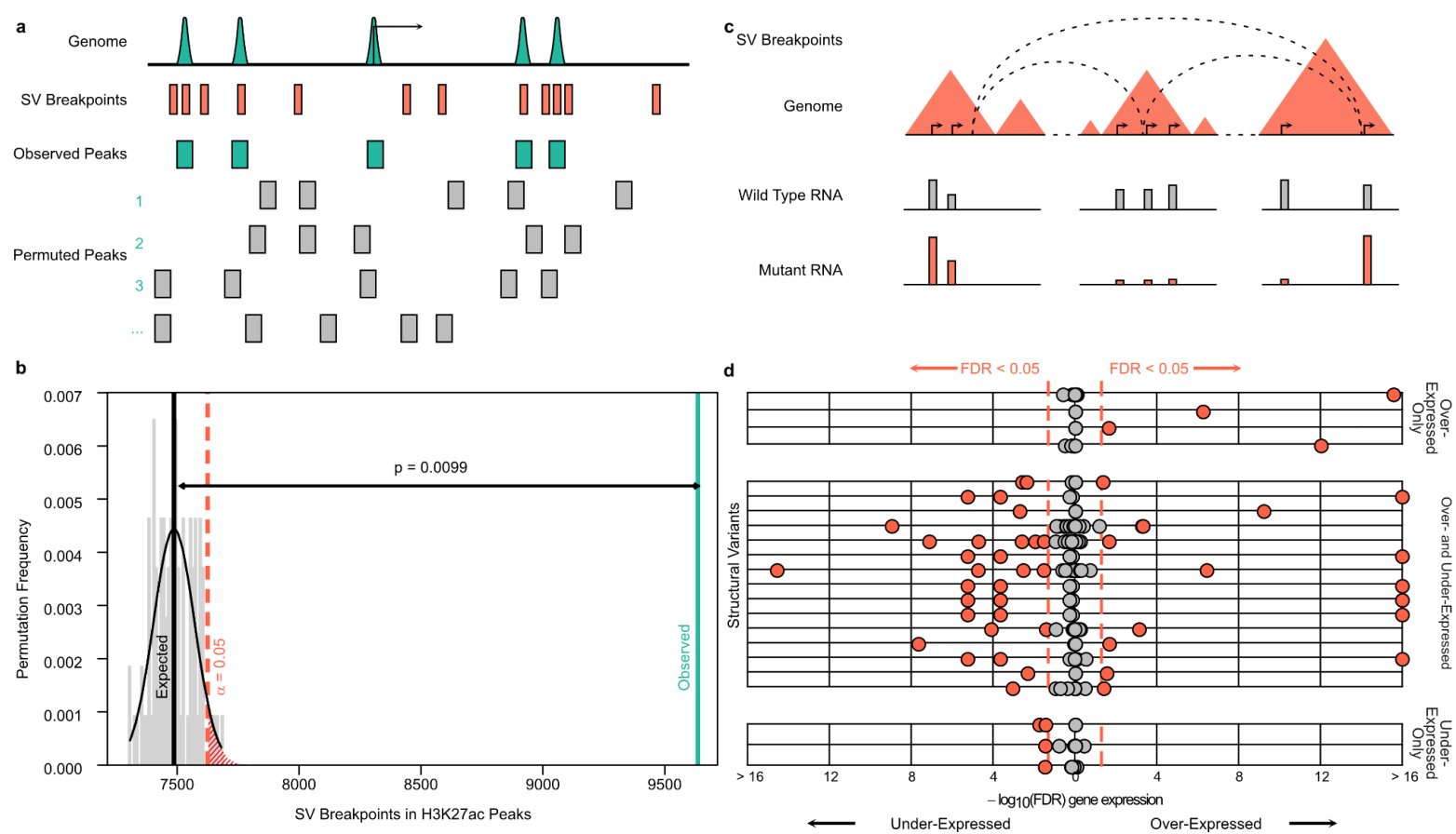

SV breakpoints are enriched in active CREs and repeatedly alter the expression of

multiple genes. a. Schematic of permutation testing for the overlap between SV breakpoints in all CPC-GENE prostate tumours and the catalogue of active CREs in the 12 tumour samples in this study. b. Histogram of permutation test results in grey. The vertical black and green bars refer to the expected and observed overlap of SV breakpoints and CREs, respectively. P-value is obtained from the permutation test, $n=100$. c. Schematic of how the expression of genes within TADs containing SV breakpoints are compared between mutant and wild type tumours are compared. d. Scatterplot of FDR values obtained from differential gene expression analysis as outlined in c. Red dots are differentially expressed genes (FDR < 0.05), grey dots are genes not differentially expressed between the mutant and wild type tumours. 


\section{Figure 6}

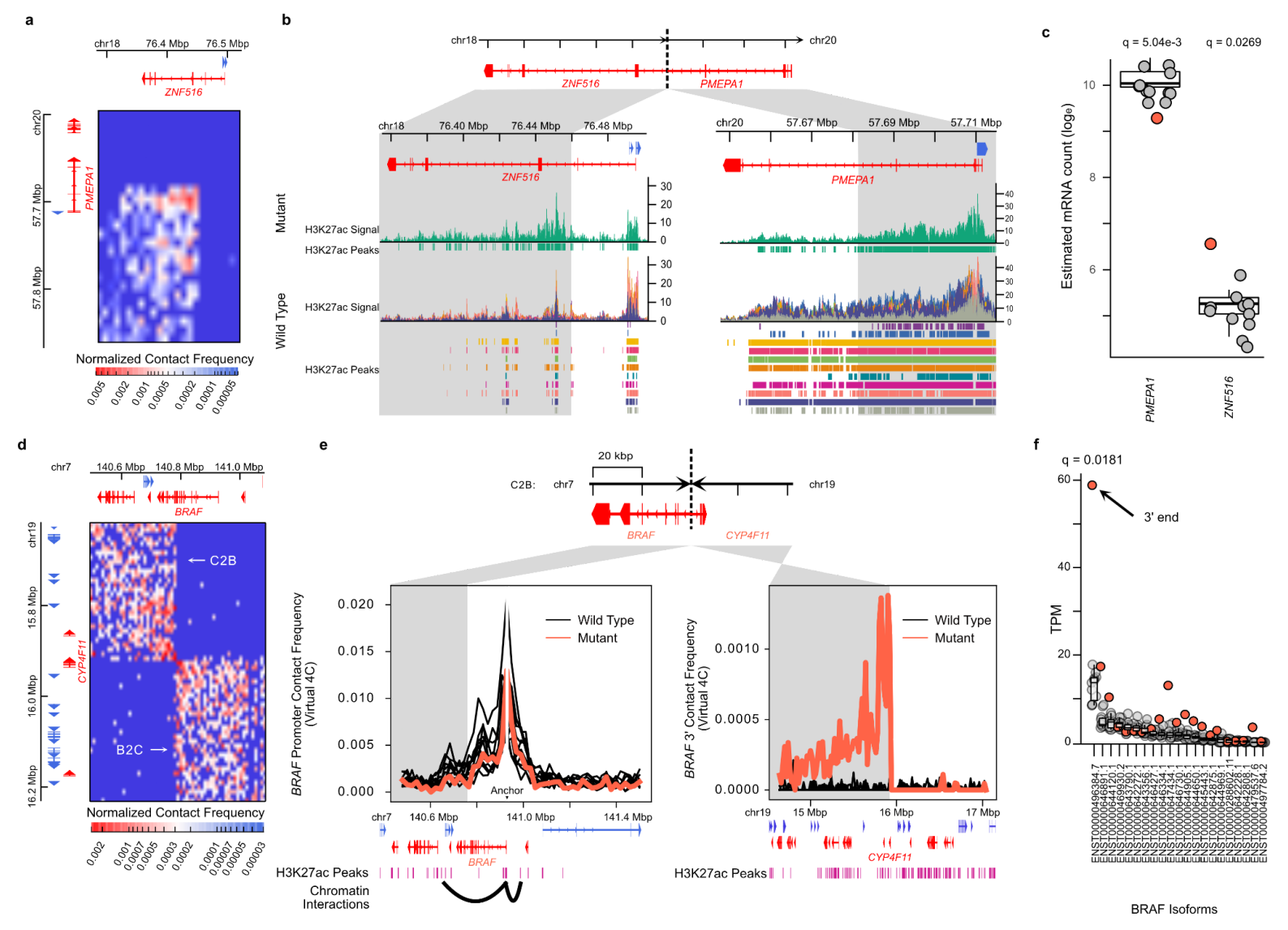

SVs altering gene expression by rewiring focal chromatin interactions. a. The contact

matrix of the deletion between PMEPAI and ZNF516. b. Genome tracks of H3K27ac ChIP-seq signal around the ZNF516 and PMEPA1 genes with the rearrangement. Grey regions are loci brought into contact by the SV. c. Gene expression of PMEPA1 and ZNF516 in all tumour samples. Boxplots represent the first, second, and third quartiles of wild type patients (grey dots). Red dots are the gene expression for the mutated patient. d. The contact matrix of an inter-chromosomal break between chromosome 7 and chromosome 19. e. Contact frequencies of the $B R A F$ promoter on chromosome 7 (left) and the 3' end of $B R A F$ on chromosome 19 (right). SV-associated contacts between the 3' end of BRAF on chromosome 19 (right) are focally enriched at H3K27ac peaks downstream of CYF4P11. Bar plot of SVs categorized by how differentially expressed genes altered. 
bioRxiv preprint doi: https://doi.org/10.1101/2021.01.05.425333; this version posted April 27, 2021. The copyright holder for this preprint (which was not certified by peer review) is the author/funder, who has granted bioRxiv a license to display the preprint in perpetuity. It is made available under aCC-BY-NC 4.0 International license. 


\section{Supplementary Figures}

\section{Supplementary Figure 1}
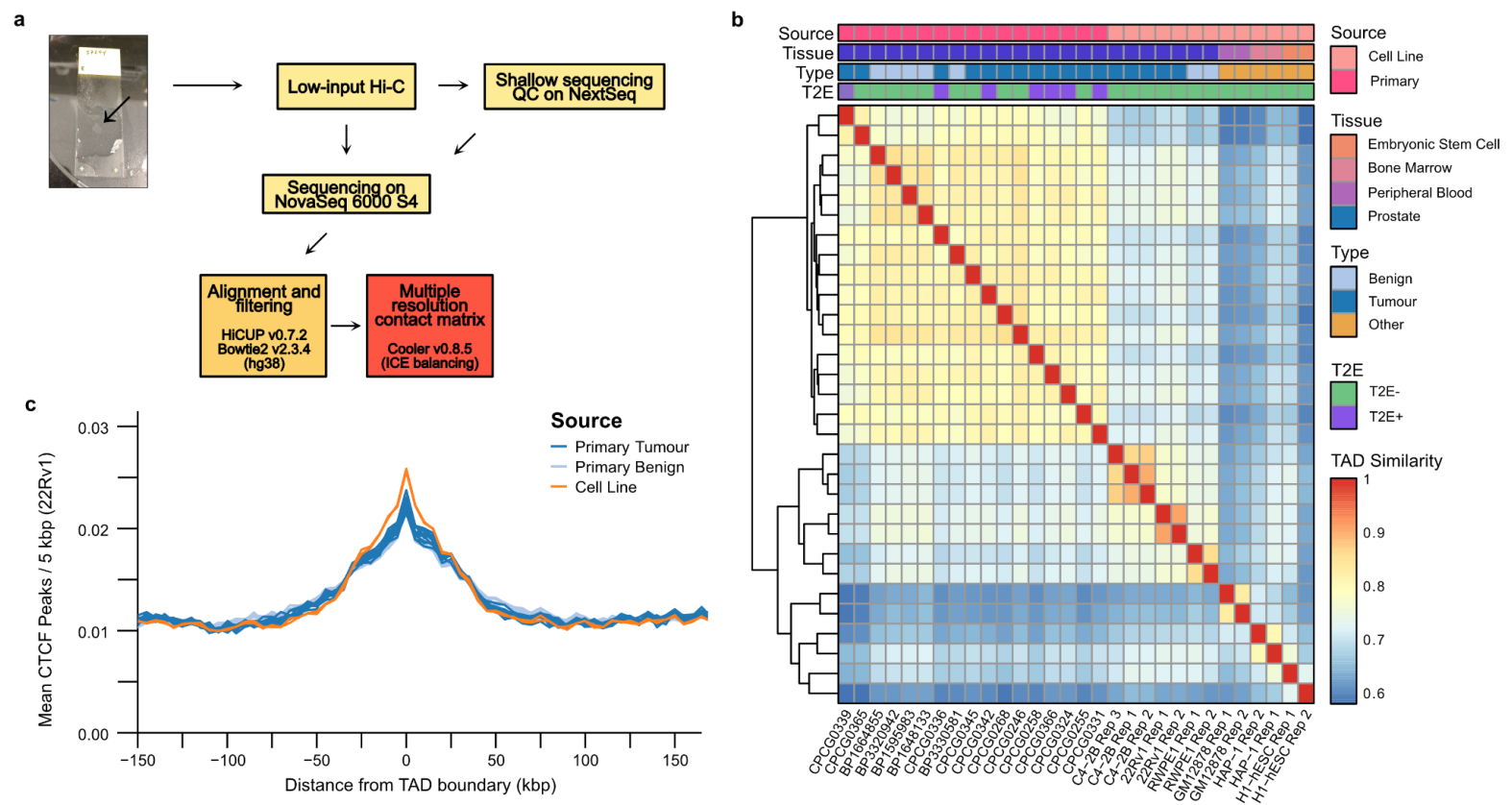

Sample processing and TAD similarity between samples. a. Schematic representation of the protocol and data pre-processing pipeline used in this study to obtain $\mathrm{Hi}-\mathrm{C}$ sequencing data. b. Heatmap of TAD similarities between primary prostate samples, prostate cell lines, and non-prostate cell lines. Median similarity scores between TADs in primary prostate tissues and cell lines is $72.1 \%, 66.9 \%$ between prostate and non-prostate cell lines, and $63.5 \%$ between primary prostate and non-prostate lines. c. Local enrichment of CTCF binding sites from the 22Rv1 PCa cell line around TAD boundaries identified in the primary samples. 
bioRxiv preprint doi: https://doi.org/10.1101/2021.01.05.425333; this version posted April 27, 2021. The copyright holder for this preprint (which was not certified by peer review) is the author/funder, who has granted bioRxiv a license to display the preprint in perpetuity. It is made available under aCC-BY-NC 4.0 International license.

\section{Supplementary Figure 2}

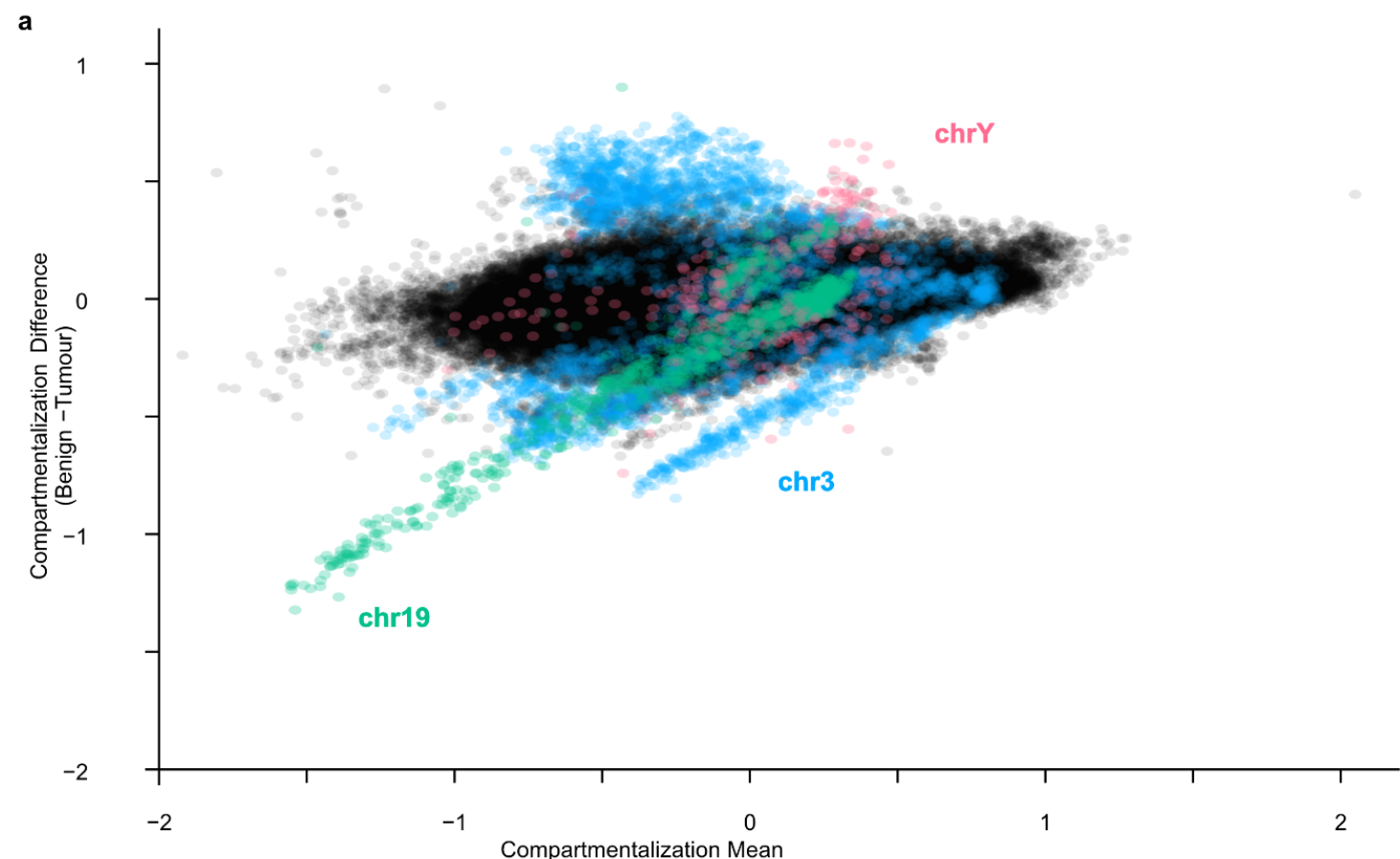

b
chr19

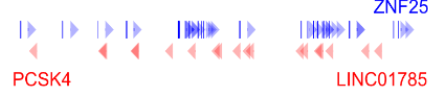

Benign Compartment E1

and in

Tumour Compartment E1

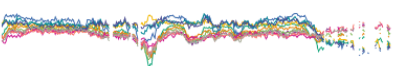

Mbp $40 \mathrm{Mbp}$ $50 \mathrm{Mbp}$

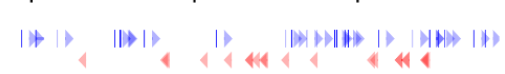

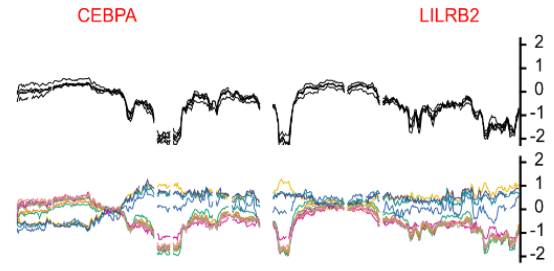

d

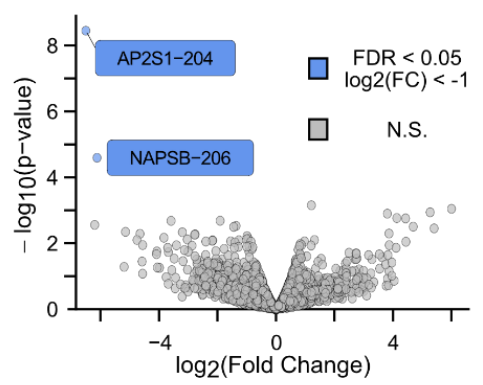

C

\begin{tabular}{llllll} 
chrY & $5 \mathrm{Mbp}$ & & $10 \mathrm{Mbp}$ & $15 \mathrm{Mbp}$ & $20 \mathrm{Mbp}$ \\
\hline CSF2RA & & & TSPY3 & VCY & HSFY2
\end{tabular}

Benign Compartment E1
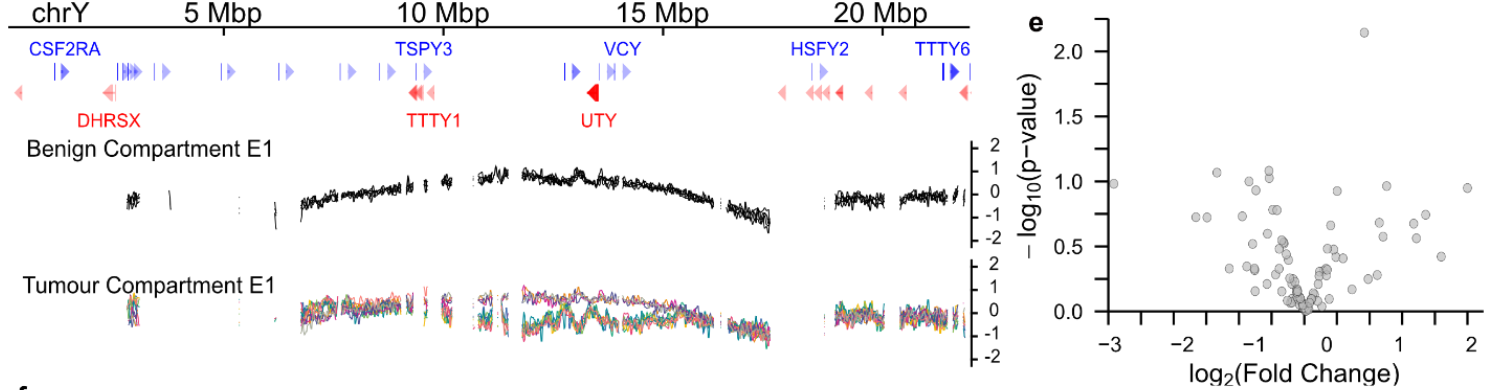

f

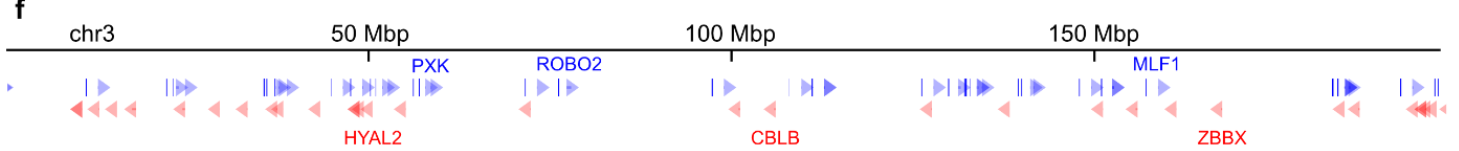

Benign CompartmentE1 


\section{Compartmentalization changes in tumours is not associated with widespread}

differential gene expression. a. Bland-Altman plot of the mean compartmentalization score between tumour and benign samples. Chromosomes 3, 19, and $\mathrm{Y}$ are highlighted for their consistent deviation between the tissue types. b-c. Compartmentalization genome tracks across chromosomes 19 (b) and Y (c) in all primary samples. d-e. Volcano plot of differential transcript expression between the tumour samples with benign-like compartmentalization and altered compartmentalization in chromosomes 19 (d) and Y (e). Grey dots are transcripts without significant differential expression, blue dots are differentially expressed transcripts $($ FDR $<0.05)$ that are under-expressed in the altered compartment samples. f. Compartmentalization genome tracks across chromosome 3. 


\section{Supplementary Figure 3}

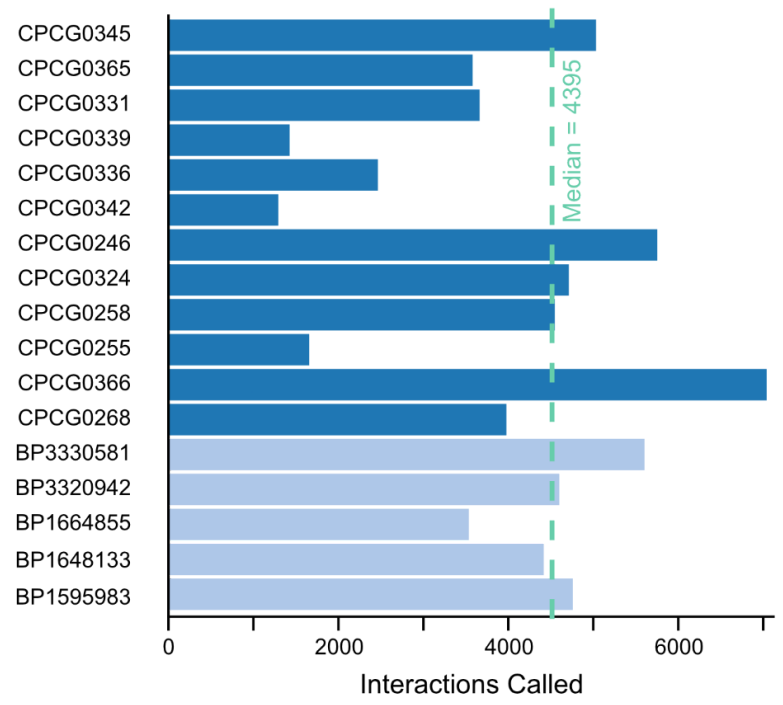

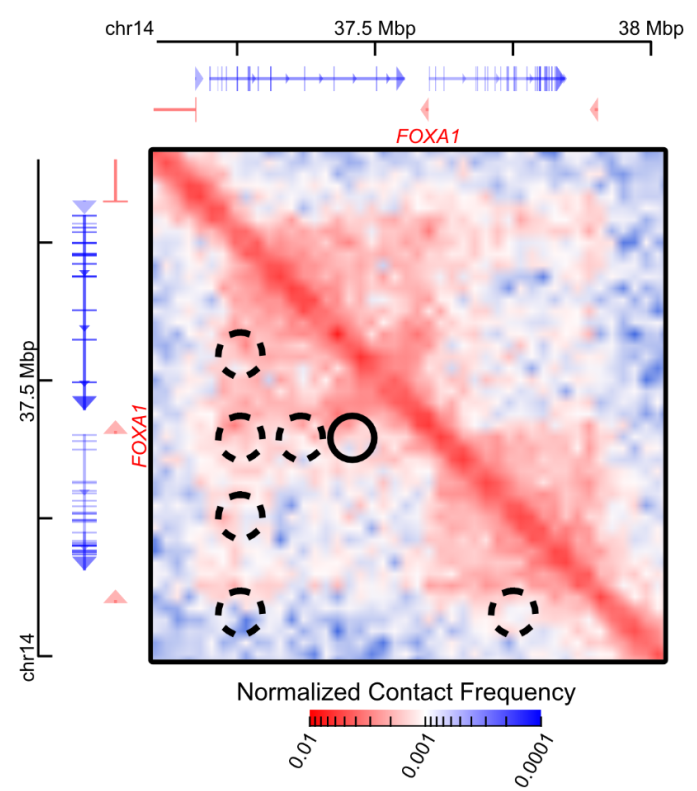

c

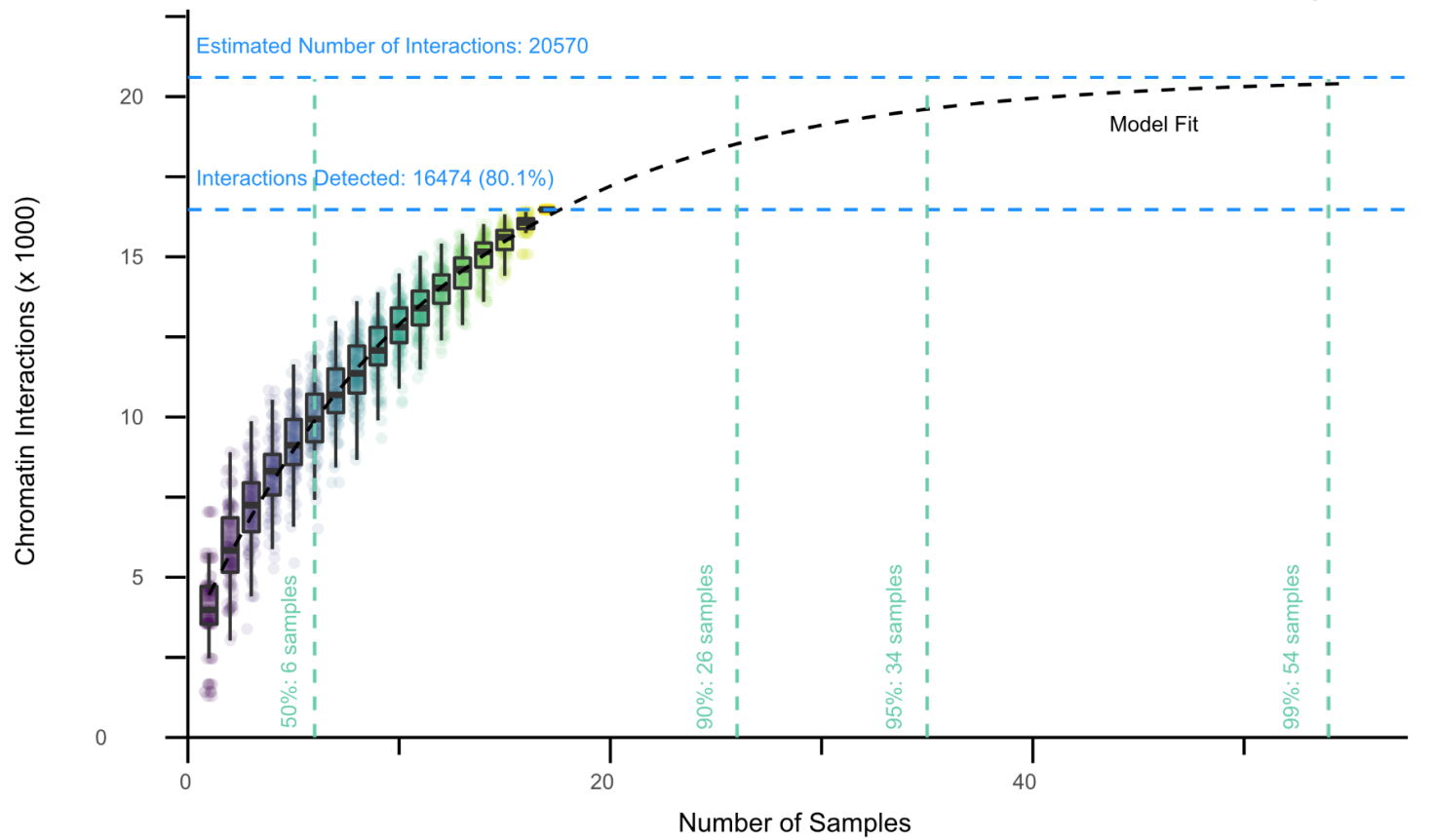

Characterization of chromatin interactions in benign and tumour tissue.

a. Barplot of the number of significant chromatin interactions identified in each of the primary prostate samples. b. A snapshot of significant chromatin interactions called around the FOXAl gene. Identified interactions are highlighted as circles. The interaction marked by the solid border contains two CREs of FOXA1 identified in $\mathrm{REF}^{19}$ (listed in that publication 
as CRE1 and CRE2). The interactions marked by the dashed border indicate regions of increased contact that may contain more distal CREs of FOXA1. c. Saturation analysis chromatin interactions detected in our cohort of prostate samples versus the theoretical estimation obtained through asymptotic estimation from bootstraps. Boxplots show the first, second, and third quartiles of the identified interactions across the bootstrap iterations. The dashed black line corresponds to the asymptotic model of estimated mean unique interactions obtained from an increasing number of samples. Horizontal blue dashed lines indicate the number of observed unique interactions and theoretical maximum. Vertical green dashed lines indicate the number of samples required to reach as estimated $50 \%, 90 \%, 95 \%$, and $99 \%$ of the theoretical maximum. 


\section{Supplementary Figure 4}
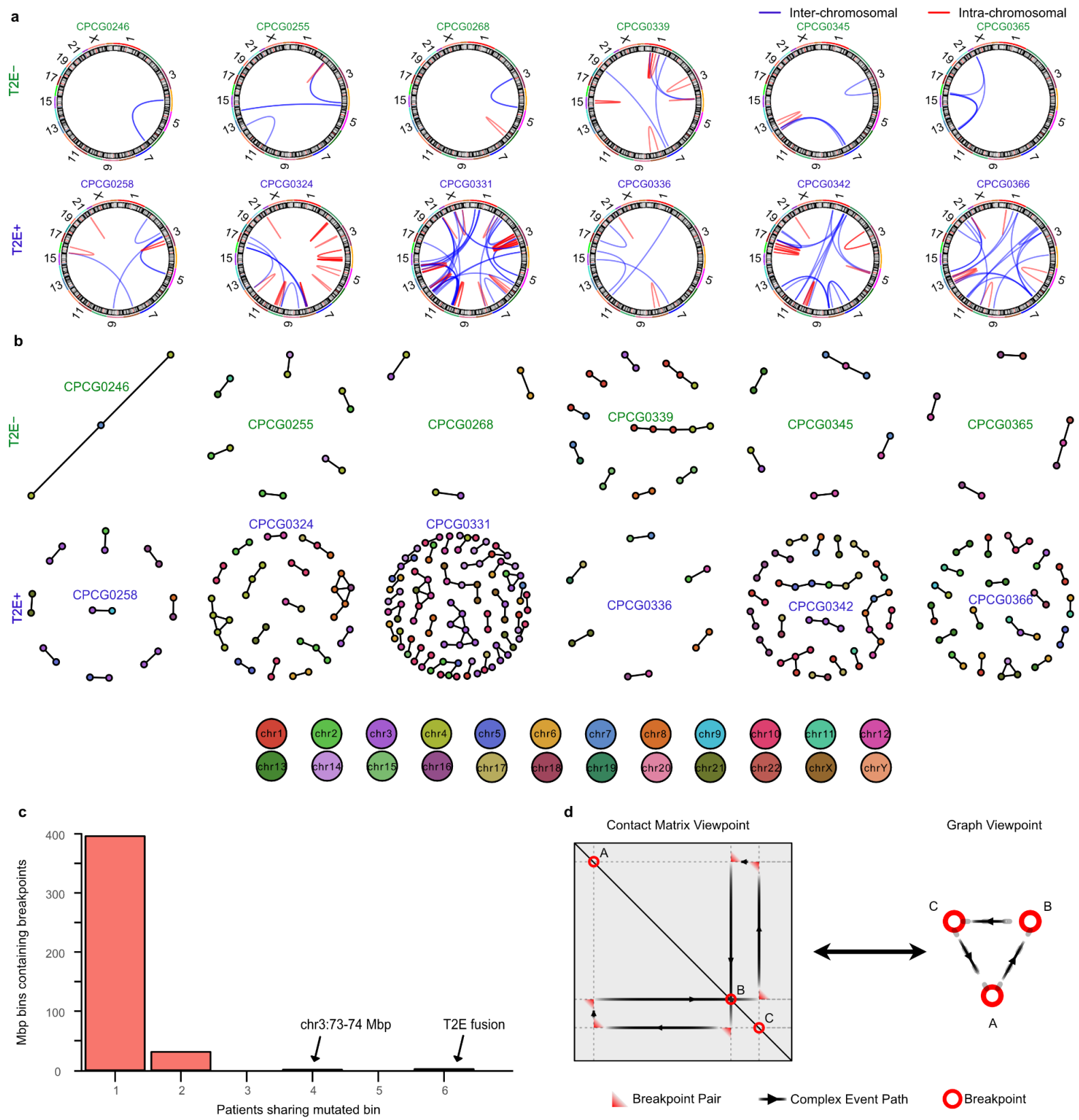

Structural variant detection from Hi-C data. a. Circos plots of structural variants identified in the 12 primary prostate tumours. b. Graph reconstructions of the simple and complex SVs in all 12 tumours. The node colour corresponds to the chromosome of origin. The nodes are spaced by a spring-force layout which is then adjusted using the Kambda Kawai optimization ${ }^{90}$ from the NetworkX Python package ${ }^{91}$. c. Barplot of the number of 1 Mbp bins with SV breakpoints from multiple patients. The previously-reported 

made available under aCC-BY-NC 4.0 International license.

highly-mutated regions on chr3 and T2E fusion are highlighted. d. Correspondence between the breakpoint representation in the contact matrices and a graph representation. Each node represents a breakpoint and each edge determines whether the breakpoints were directly in contact, as identified by the Hi-C contact matrix. 


\section{Supplementary Figure 5}

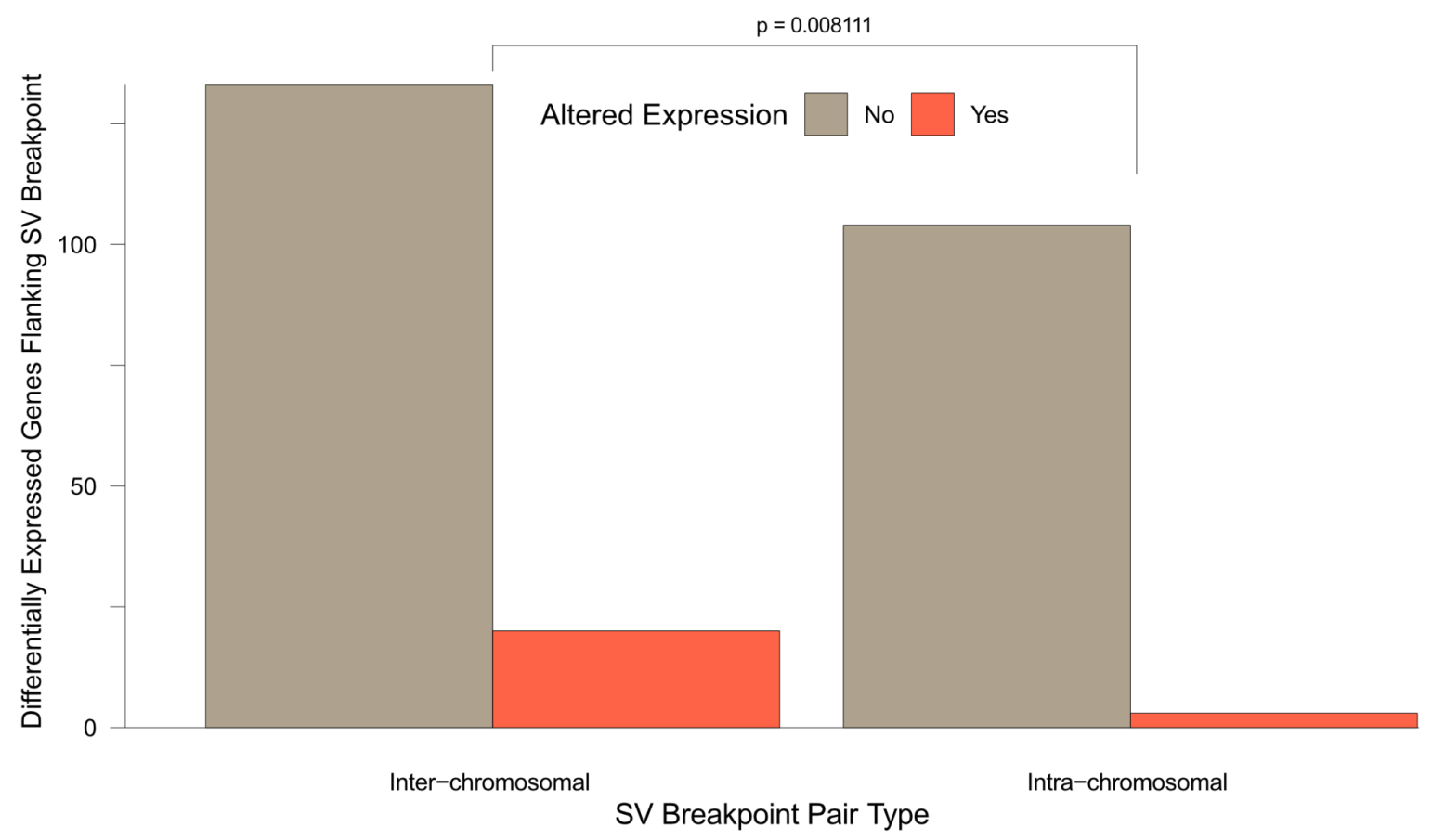

Relationship between inter-chromosomal rearrangements and differential gene

expression. Barplot of the number of differentially expressed genes and whether they are involved in SVs spanning multiple chromosomes. Pearson's chi-squared test, $X^{2}=7.0088, p$ $=0.00811, \mathrm{df}=1$. 


\section{Supplementary Figure 6}

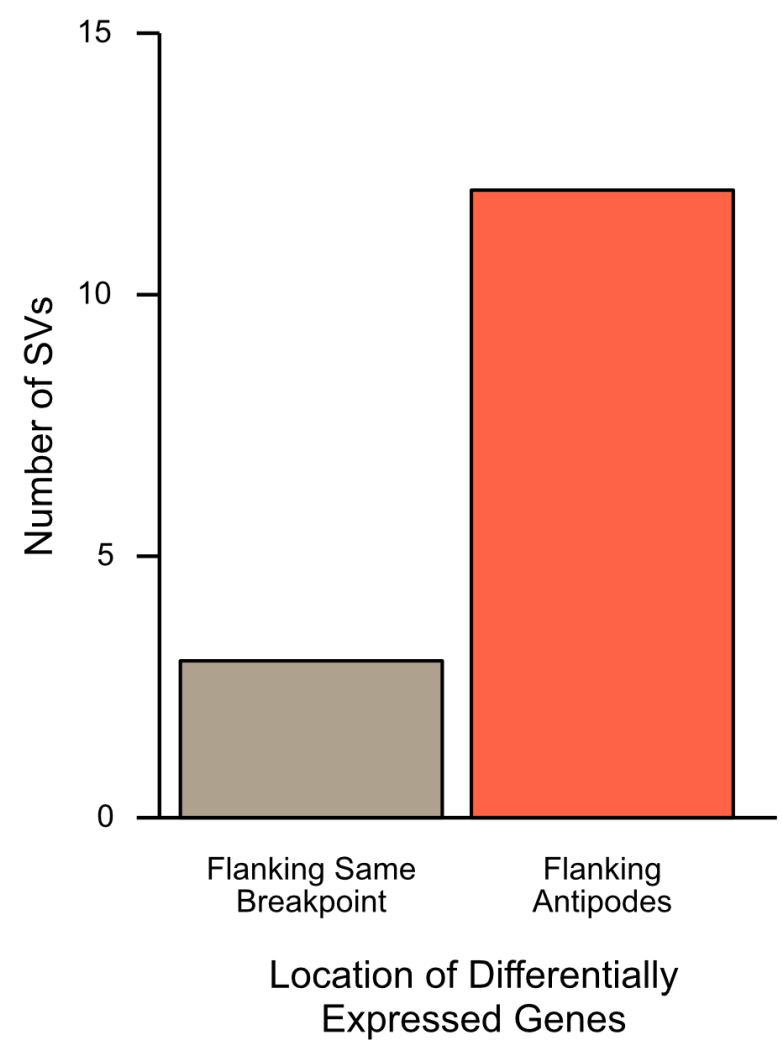

Location of differentially expressed genes around SV breakpoints. Barplot of all 15 SVs

associated with both over- and under-expression, categorized by which breakpoints the differentially expressed genes flank. 


\section{Supplementary Figure 7}
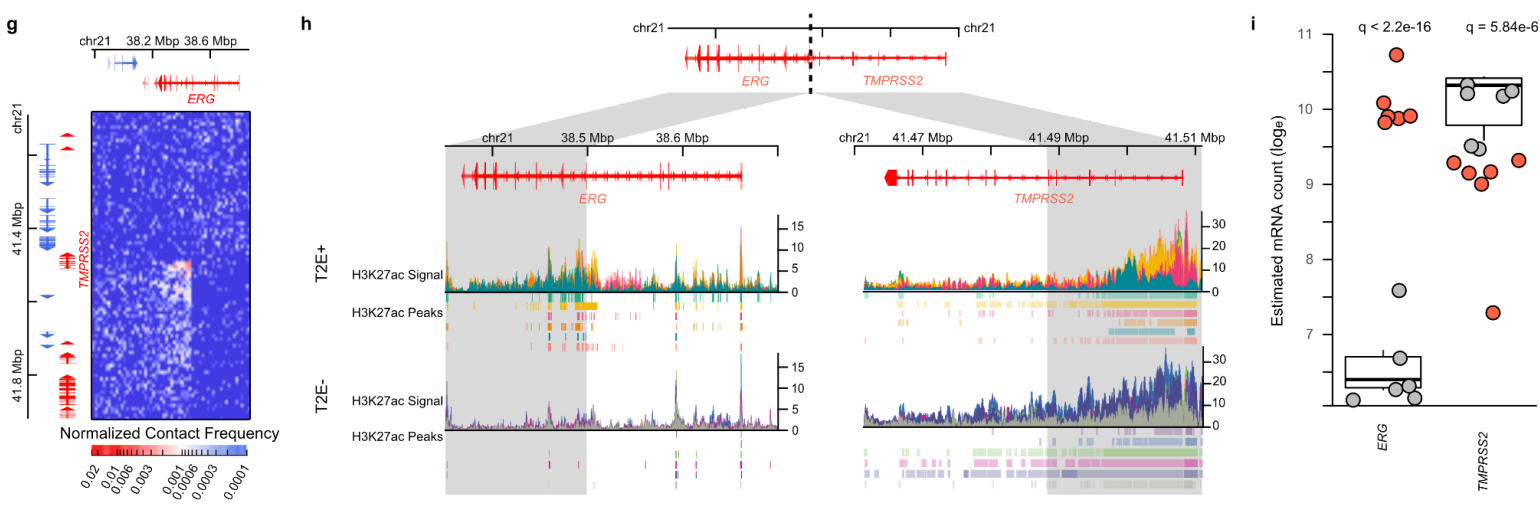

Chromatin organization of the TMPRSS2-ERG fusion. a. Contact matrix of the deletion between TMPRSS2 and ERG. b. Genome tracks of H3K27ac ChIP-seq signal in T2E+ and T2E- patients. The grey region highlights the loci that come into contact as a result of the deletion. c. Expression of TMPRSS2 and ERG genes. Boxplots represent first, second, and third expression quartiles of T2E- patients (grey dots). T2E+ patients are represented by red dots. 


\section{Supplementary Tables}

Supplementary Table 1 - Clinical information of samples involved in this study.

Supplementary Table 2 - Sequencing metrics as calculated by HiCUP for all Hi-C libraries generated in this study.

Supplementary Table 3 - Summary statistics for TAD counts in all 12 tumour and 5 benign samples, across multiple window sizes.

Supplementary Table 4 - Individual TAD calls in all 12 tumour and 5 benign samples.

Supplementary Table 5 - Detected chromatin interactions in all 12 tumour and 5 benign samples.

Supplementary Table 6 - SV breakpoints detected by Hi-C in each tumour sample.

Supplementary Table 7 - Simple and complex SVs reconstructed from SV breakpoints.

Supplementary Table 8 - H3K27ac peaks identified in each of the 12 primary PCa patients.

Raw sequencing data as previously published in ${ }^{42}$ was remapped to the hg38 reference genome. 